\title{
Set of Agricultural Land Evaluation in El-Dakhla \\ Oases Soils, Egypt
}

Soliman, M.M.

Soils, Water and Environment. Res. Inst., Agric. Res. Center

L-DAKHLA Oases soils are located between longitudes $28^{\mathrm{O}} 30^{-}$
${\text {and } 29^{\circ}}^{\circ} 04^{-}$East and latitudes $25^{\circ} 20^{-}$and $26^{\circ} 00^{-}$North, covering about $2000 \mathrm{~km}^{2}$.

Physiographic mapping units, soil taxonomy, statistical size parameters, water resources quality and its suitability for irrigation and crop water requirements were performed as a set of agricultural land capability and suitability evaluation of El- Dakhla Oases soils.

Land sat ETM image (2010), digital elevation model (DEM) and 3D -GIS techniques were used in ERDAS image 9.2 software to produce the physiographic map of the studied area. The main physiographic units and its soil taxonomic ; family levels could be classified as follows:

1- Soils of Playa:

a- Typic Haplosalids, fine loamy over sandy skeletal, mixed, hyperthermic.

b- Duric Haplosalids, sandy skeletal, mixed, hyperthermic.

2-Soils of Sabkha:

Lithic Gypsisalids, sandy skeletal, siliceous, hyperthermic, shallow.

3- Soils of Sand Sheets:

Calcic Haplosalids, sandy skeletal, mixed, hyperthermic, deep.

4- Soils of Peniplain:

Typic Haplosalids, clayey skeletal, hyperthermic.

5- Soils of Cultivated plain:

a- Typic Torriorthents, fine loamy, mixed, hyperthermic.

b-Typic Torriorthents, clayey over fine loamy skeletal, mixed, hyperthermic.

c-Typic Haplosalids, coarse loamy skeletal over clayey, mixed, hyperthermic .

d-Typic Haplosalids, fine loamy over coarse loamy, mixed, hyperthermic.

The statistical size distribution reveal that these soils have mainly poorly sorted sediments with near symmetrical to fine skewed materials and lepto kurtic to meso kurtic pattern. These parameters indicate that the studied area is formed under water or both water and wind action, forming of non- uniform parent materials.

Current suitability of the studied soils could be categorized into three suitable classes; moderately suitable $\left(\mathrm{S}_{2}\right)$, marginally suitable

E-mail:mahmoudaldemelawi@hotmail.com

:mahmoudeldemelawi@yahoo.com 
$\left(\mathrm{S}_{3}\right)$ and not suitable $\left(\mathrm{N}_{1}\right)$ with different intensity degree of soil limitations. By existing suitable improvement practices, the potential suitability classes assessed are two suitability classes; moderately suitable $\left(\mathrm{S}_{2}\right)$ and not suitable $\left(\mathrm{N}_{2}\right)$.

Data revealed that current suitability for some specific crops were not suitable $(\mathrm{N})$, except for some scattered areas developed on cultivated plain which are suitable for all the studied crops. On the other hand, the potential suitability classes differed according to the satisfaction conditions between different properties of soils developed on the studied physiographic units and crop water requirements. However, soils of sabkha and cultivated plain are highly suitable $\left(S_{1}\right)$ for groundnut. Also, soils of peniplain and cultivated plain are highly suitable $\left(\mathrm{S}_{1}\right)$ for potato and grapes. Whereas, soils of playa, peniplain and cultivated plain are moderately suitable $\left(\mathrm{S}_{2}\right)$ for olives.

Water of wells and springs are considered the main sources for irrigation in El- Dakhla Oases soils. Data revealed that the suitability of irrigation water had $\left(\mathrm{C}_{1}-\mathrm{S}_{1}\right)$ class with non restriction at west El-Mowhob and Kalamon area , while it represented $\left(\mathrm{C}_{4}-\mathrm{S}_{4}\right)$ class with very high salinity levels and very high alkalinity hazard at El-Zaiate well and Bathor spring waters, indicating severe restrictions in these soils.

Data revealed that the crop water requirement values of some specific crops are considered high, due to the highness of evapotranspiration values. It represented with an average of 500, 800 and $1800 \mathrm{~mm} / \mathrm{s}$ for vegetable, field and fruit crops, respectively. So, it is very important to apply suitable irrigation systems such as trickle or sprinkler, under these conditions.

Keywords: GIS, Land evaluation, Soil taxonomy, and Crop water requirements.

El- Dakhla depression is natural excavation in the middle part of the Egyptian Western Desert and has an area of about $2000 \mathrm{~km}^{2}$. It is situated between latitudes $25^{\circ} 20^{\circ}$ and $26^{\circ} 00^{-}$North and Longitudes $28^{\circ} 30^{\circ}$ and $29^{\circ} 04^{-}$East, It is located at about $120 \mathrm{~km}$ west of El- Kharga Oases and about $300 \mathrm{~km}$ west the Nile Valley. The floor of El- Dakhla depression is bound from the north and north east by steep scarps of the Eocene limestone plateau, but gradually rises to the south where it merges with the plain of upper Cretaceous Nubian sandstone.

\section{Geology}

A number of geologists, among them Shata (1959), Said (1962), Hermina (1967), Attia (1970), Abu El- Izz (2000) and Said (2000) discussed the geological description of El- Dakhla Oases. They mentioned that the succession of its formation from the olders to the youngest, i.e. Cretaceous, Paleocene and Quaternary (Pleistocene and Holocene). It owes its orgin to the exposure of Nubian sediments which consist of alternation of clays, shales, sands and sandstones to erosion. 
The overlying Cretaceous and Eocene formations consist essentially of limestone from the plateau which borders the Oasis from north and east, the elevation of this plateau indicates that erosion has removed about $200 \mathrm{~m}$ of this limestone to expose the underlying Nubian rocks. The latter group rocks contains the important water bearing horizon of which the oasis owes its existence.

\section{Climate}

According to Central Laboratory for Agriculture of Climate (CLAC 2010), the climatelogical data of El- Dakhla Oases is somewhat worm in summer and slightly cold in winter. The mean annual temperature ranges between $13.7^{\circ} \mathrm{C}$ and $33^{\circ} \mathrm{C}$, the annual maximum temperature differs from $21.2^{\circ} \mathrm{C}$ to $40.6^{\circ} \mathrm{C}$ and the minimum from $6.2^{\circ} \mathrm{C}$ to $25.7^{\circ} \mathrm{C}$. El- Dakhla Oases receives a very low amount of rainfall where the average rate is located between 0.1 and $1.2 \mathrm{~mm} / \mathrm{year}$. The mean annual relative humidity ranges from $28.0 \%$ to $66 \%$. Wind velocity ranges from $5.2 \mathrm{Km} / \mathrm{h}$ in December to $19.2 \mathrm{Km} / \mathrm{h}$ in March. According to the Key of Soil Taxonomy System (USDA 2010) the soil temperature regime of the studied area could be defined as hyperthermic and soil moisture regime as torric.

\section{Water resources}

In El- Dakhla Oases there are two main sources of irrigation waters, water of springs and water of wells. Most wells of El- Dakhla Oases are deep, their sources is the Nubian Sandstone which is saturated with water originating from equatorial rainfall, water is found at varying depths, between 300 and $400 \mathrm{~m}$. Some geologists think that ground water movement from Equatorial Africa to the Western Desert for about 500 years.

\section{Material and Methods}

Based on the interpretation of the remote sensing image of El- Daklla Oases, the following geomorphic aspects were distinguished:

1- Structural Plateau (SP)

2- Escarpment (ES)

3- Peniplain (Pe)

3- 1- Cultivated area $(\mathrm{Cu})$

3-2- Playa $(\mathrm{Pe} \mathrm{Pa})$

3-3- Sabkha (Pe sb)

4- Wind blown sand (W)

4-1- Sand dunes (Wsd)

4-2-Sand sheets (Wss)

Table 1 shows the geomorphic legend description of different mapping units. Figure 1 illustrates physiographic units of El- Dakhla Oases delineated on landsat, Thematic mapper hard copy (2010) produced by ERDAS imagine 9.3 image processing software. Topographic maps scale $1: 100.000$, produced by Egyptian General Survey Authority were used to check the delineated soil units of the study area. 


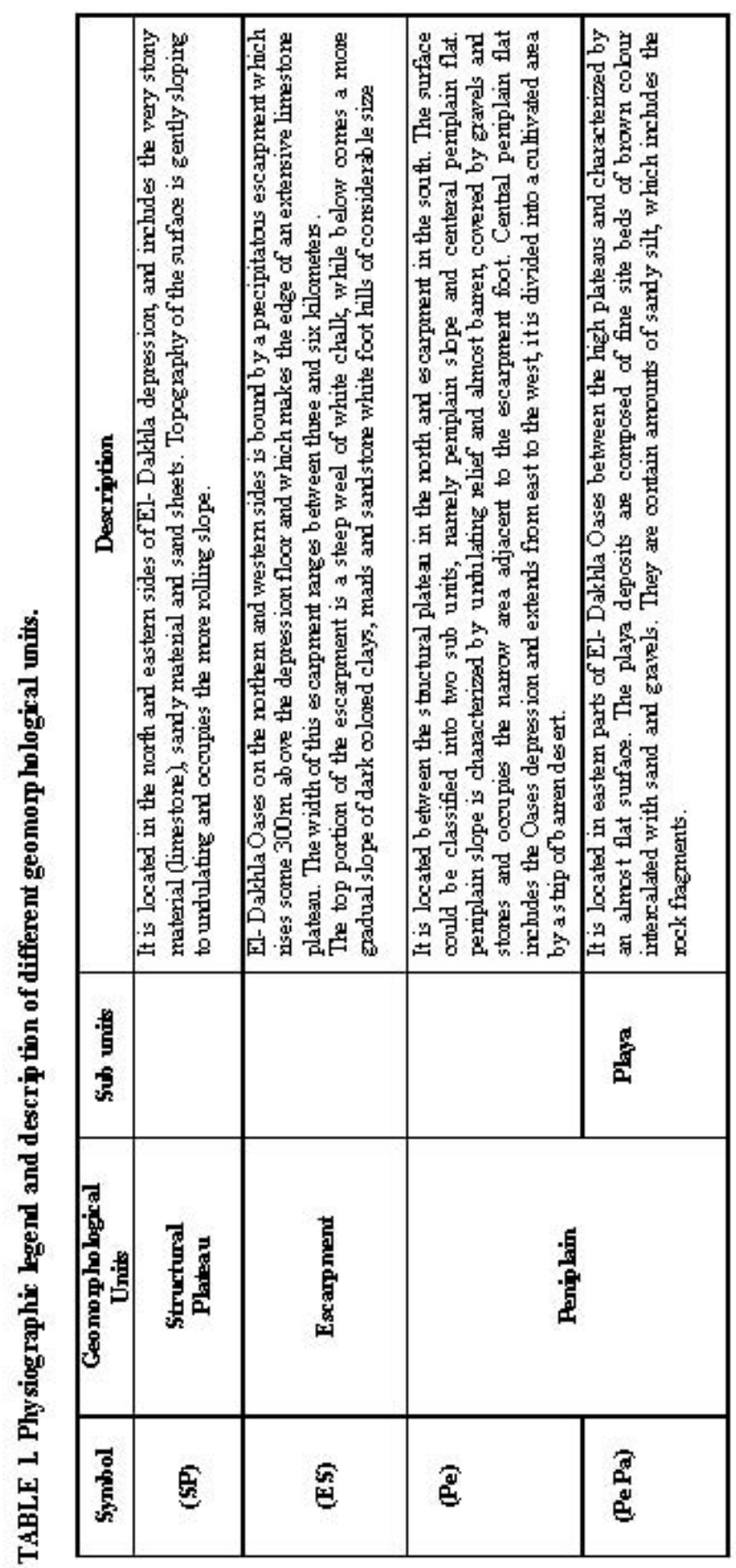

Egypt. J. Soil Sci. 56, No. 1 (2016) 
SET OF AGRICULTURAL LAND EVALUATION IN EL-DAKHLA ... 5

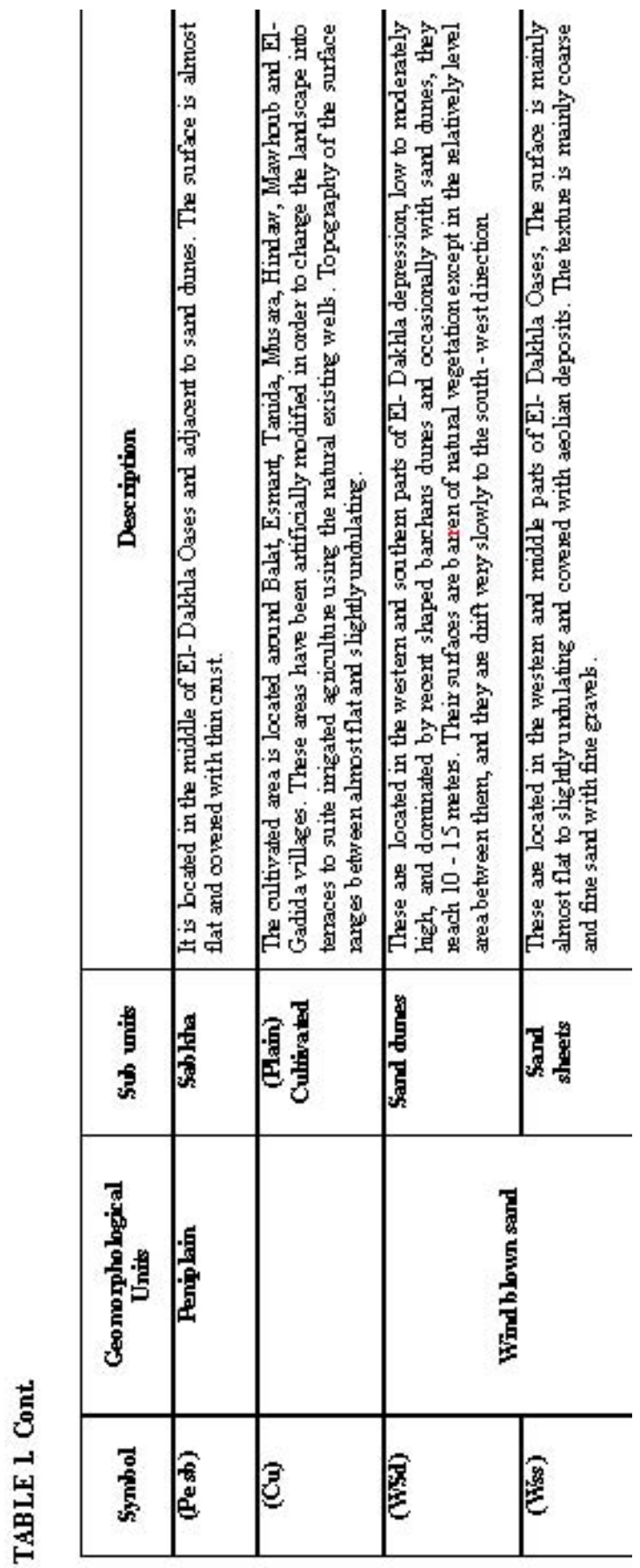

Egypt. J. Soil Sci. 56, No.1 (2016) 


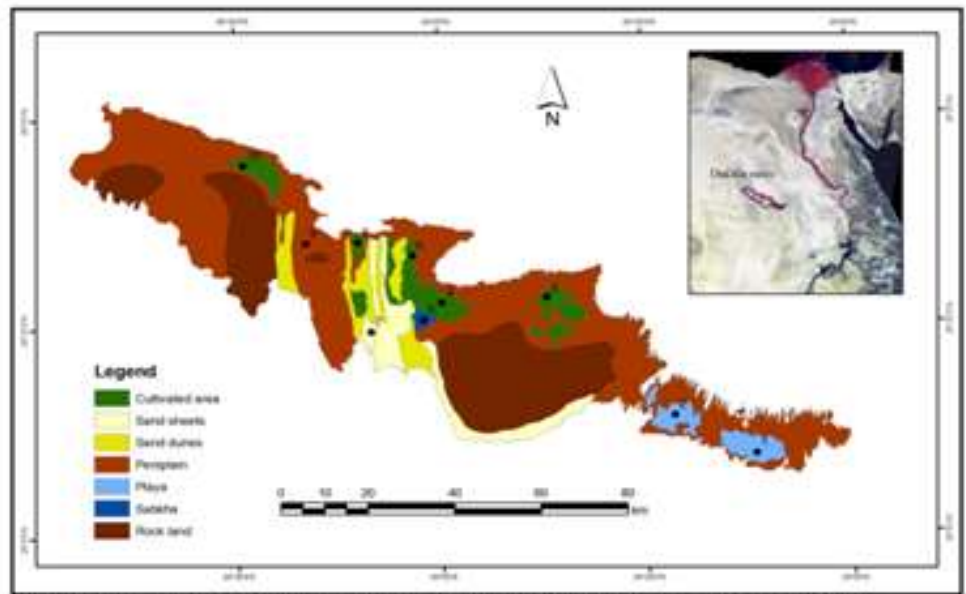

Fig. (1): Physiographic Units and profiles locations in E.I Dakhla Oasis.

\section{Field work}

A rapid reconnaissance soil survey was made throughout the investigated area of El- Dakhla Oases in order to identify the major landforms and gain appreciation of the broad soil patterns and landscape characteristics. The primary units were verified based on field interpretation and information gained during the reconnaissance survey. Ten soil profiles have been dug in each site to a depth of about $150 \mathrm{~cm}$ or to the depth of hard layers, parent material, or the water table. A detailed morphological description of soil profiles was carried out on basis outlined by FAO (2006), (Table 2). A number of 37 soil samples of the various layers have been collected for laboratory analyses. (Fig.1). Also, ten water samples representing the wells and springs were collected.

\section{Laboratory Analyses}

Physical analyses

Soil color (wet \& dry) was identified with the aid of Munsell (2010). Mechanical analysis was carried out for fraction by pipette method and particle size distribution for sand fraction was determined by dry sieving USDA (2004), then the obtained data were statistically evaluated according to Folk and Ward (1957).

\section{Chemical analyses}

Electrical conductivity (EC), $\mathrm{PH}$, soluble cations and anions, $\mathrm{CaCO}_{3} \%$, OM\%, and gypsum contents were determined according to USDA (2004). Ten water samples were subjected to chemical analyses according to USDA (2004), where soluble cations and anions, total dissolved salts (TDS) and $\mathrm{pH}$ were determined. Sodium adsorption ratio (SAR) was calculated using the formula :

$$
S A R=\frac{\mathrm{Na}^{+}}{\sqrt{\frac{\mathrm{Ca^{++ } + M g ^ { + + }}}{2}}}
$$

Egypt. J. Soil Sci. 56, No. 1 (2016) 
TABLE 2. Morphological Description of the Studied Soil Profiles.

\begin{tabular}{|c|c|c|c|c|c|c|c|c|c|c|}
\hline \multirow{2}{*}{ 递 } & \multirow{2}{*}{$\begin{array}{c}\text { Depth } \\
\text { (cm) }\end{array}$} & \multicolumn{4}{|c|}{ Soil colour } & \multirow{2}{*}{ 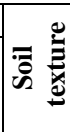 } & \multirow{2}{*}{ 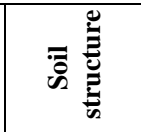 } & \multirow[b]{2}{*}{ Consistence } & \multirow{2}{*}{ 绨芯 } & \multirow{2}{*}{ 胥 } \\
\hline & & \multicolumn{2}{|c|}{ Dry } & \multicolumn{2}{|c|}{ Moist } & & & & & \\
\hline \multicolumn{11}{|c|}{ Playa } \\
\hline 1 & $\begin{array}{c}0-30 \\
30-60 \\
60-80 \\
80-150\end{array}$ & $\begin{array}{l}5 \mathrm{YR} \\
5 \mathrm{YR} \\
5 \mathrm{YR} \\
5 \mathrm{YR}\end{array}$ & $\begin{array}{l}5 / 3 \\
5 / 4 \\
5 / 4 \\
5 / 4\end{array}$ & $\begin{array}{l}5 \mathrm{YR} \\
5 \mathrm{YR} \\
5 \mathrm{YR} \\
5 \mathrm{YR}\end{array}$ & $\begin{array}{l}5 / 4 \\
4 / 6 \\
4 / 6 \\
4 / 4\end{array}$ & $\begin{array}{l}\text { SCL } \\
\text { SCL } \\
\text { LS } \\
\text { SL }\end{array}$ & $\begin{array}{l}\text { w.c.s.ang } \\
\text { m.c.ang b } \\
\text { ma } \\
\text { ma } \\
\text { ma }\end{array}$ & $\begin{array}{l}\text { soft nst npl } \\
\text { soft nst npl } \\
\text { soft nst npl } \\
\text { soft nst npl }\end{array}$ & $\begin{array}{l}\text { Non } \\
\text { Non } \\
\text { Non } \\
\text { Non }\end{array}$ & $\begin{array}{l}\text { c.s } \\
\text { c.s } \\
\text { c.s } \\
--\end{array}$ \\
\hline 2 & $\begin{array}{c}0-10 \\
10-50\end{array}$ & $\begin{array}{l}7.5 \mathrm{YR} \\
7.5 \mathrm{YR}\end{array}$ & $\begin{array}{l}5 / 4 \\
5 / 3\end{array}$ & \begin{tabular}{l|}
$7.5 \mathrm{YR}$ \\
$7.5 \mathrm{YR}$ \\
\end{tabular} & $\begin{array}{l}4 / 4 \\
4 / 3\end{array}$ & $\begin{array}{l}\text { SCL } \\
\text { LS }\end{array}$ & $\begin{array}{l}\text { w.c.s.ang } \\
\text { w.c.s.ang }\end{array}$ & $\begin{array}{l}\text { soft ns np } \\
\text { soft ns np }\end{array}$ & $\begin{array}{l}\text { W } \\
\text { W }\end{array}$ & c.s \\
\hline \multicolumn{11}{|c|}{ Sabkha } \\
\hline 5 & $\begin{array}{c}0-10 \\
10-40\end{array}$ & $\begin{array}{l}5 \mathrm{YR} \\
5 \mathrm{YR}\end{array}$ & $\begin{array}{l}4 / 6 \\
5 / 4\end{array}$ & $\begin{array}{l}5 \mathrm{YR} \\
5 \mathrm{YR}\end{array}$ & $\begin{array}{l}4 / 4 \\
4 / 4\end{array}$ & $\begin{array}{c}\text { SCL } \\
\text { S }\end{array}$ & $\begin{array}{c}\text { w.c.s.ang } \\
\text { ma }\end{array}$ & $\begin{array}{l}\text { nst npl } \\
\text { nst npl }\end{array}$ & $\begin{array}{l}\mathrm{w} \\
\mathrm{W}\end{array}$ & $\begin{array}{c}\text { c.s } \\
--\end{array}$ \\
\hline \multicolumn{11}{|c|}{ Sand Sheets } \\
\hline 7 & $\begin{array}{c}0-15 \\
15-55 \\
55-85 \\
85-110\end{array}$ & $\begin{array}{l}7.5 \mathrm{YR} \\
7.5 \mathrm{YR} \\
7.5 \mathrm{YR} \\
7.5 \mathrm{YR} \\
10 \mathrm{YR}\end{array}$ & $\begin{array}{l}6 / 4 \\
6 / 6 \\
6 / 6 \\
5 / 4 \\
5 / 6 \\
\end{array}$ & \begin{tabular}{|l|}
$.5 \mathrm{YR}$ \\
$7.5 \mathrm{YR}$ \\
$7.5 \mathrm{YR}$ \\
$7.5 \mathrm{YR}$ \\
$10 \mathrm{YR}$ \\
\end{tabular} & $\begin{array}{l}5 / 4 \\
5 / 6 \\
5 / 6 \\
4 / 3 \\
4 / 6 \\
\end{array}$ & $\begin{array}{c}\text { S } \\
\text { LS } \\
\text { S } \\
\text { S }\end{array}$ & $\begin{array}{l}\mathrm{Sg} . \\
\mathrm{Sg} . \\
\text { ma. } \\
\text { ma. }\end{array}$ & $\begin{array}{l}\text { Lo n st. npl. } \\
\text { Lo n st. npl. } \\
\text { So. n st. npl. } \\
\text { So. n st. npl. }\end{array}$ & $\begin{array}{l}\text { W } \\
\text { w } \\
\text { Non } \\
\text { Non } \\
\text { W }\end{array}$ & $\begin{array}{l}\text { c.s } \\
\text { c.s } \\
\text { c.s } \\
\text { d.s } \\
--\end{array}$ \\
\hline \multicolumn{11}{|c|}{ Peniplain } \\
\hline 9 & $\begin{array}{c}0-15 \\
15-55 \\
55-75 \\
75-110\end{array}$ & $\begin{array}{l}10 \mathrm{YR} \\
10 \mathrm{YR} \\
10 \mathrm{YR} \\
10 \mathrm{YR}\end{array}$ & $\begin{array}{l}6 / 3 \\
5 / 3 \\
4 / 4 \\
5 / 4\end{array}$ & $\begin{array}{l}5 \mathrm{YR} \\
5 \mathrm{YR} \\
5 \mathrm{YR} \\
5 \mathrm{YR}\end{array}$ & $\begin{array}{l}5 / 6 \\
4 / 4 \\
4 / 6 \\
4 / 4\end{array}$ & $\begin{array}{c}\text { SC } \\
\text { C } \\
\text { SC } \\
\text { SC }\end{array}$ & $\begin{array}{c}\text { w.c.ang b } \\
\text { m.f.ang b } \\
\text { s.f.ang b } \\
\text { w.c.ang b }\end{array}$ & $\begin{array}{l}\text { n. st. } \\
\text { st. pl. } \\
\text { n. st. } \\
\text { n. st. }\end{array}$ & $\begin{array}{c}\text { mod } \\
\text { w } \\
\text { w } \\
\text { w }\end{array}$ & $\begin{array}{l}\text { c.s } \\
\text { c.s } \\
\text { d.s } \\
--\end{array}$ \\
\hline \multicolumn{11}{|c|}{ (Plain) Cultivated area } \\
\hline 3 & $\begin{array}{c}0-10 \\
10-35 \\
35-65 \\
65-100\end{array}$ & $\begin{array}{l}7.5 \mathrm{YR} \\
7.5 \mathrm{YR} \\
5 \mathrm{YR} \\
5 \mathrm{YR}\end{array}$ & $\begin{array}{l}4 / 6 \\
5 / 6 \\
4 / 4 \\
4 / 4\end{array}$ & \begin{tabular}{|c|}
$7.5 \mathrm{YR}$ \\
$7.5 \mathrm{YR}$ \\
$5 \mathrm{YR}$ \\
$5 \mathrm{YR}$
\end{tabular} & $\begin{array}{l}4 / 6 \\
4 / 4 \\
4 / 4 \\
5 / 6\end{array}$ & $\begin{array}{l}\text { SCL } \\
\text { SCL } \\
\text { SCL } \\
\text { SCL }\end{array}$ & $\begin{array}{c}\mathrm{ma} \\
\mathrm{ma} \\
\mathrm{ma} \\
\mathrm{ma}\end{array}$ & $\begin{array}{l}\text { st pl } \\
\text { st pl } \\
\text { st pl } \\
\text { st pl }\end{array}$ & $\begin{array}{l}\text { mod } \\
\text { mod } \\
\text { mod } \\
\text { mod }\end{array}$ & $\begin{array}{l}\text { d.s } \\
\text { d.s } \\
\text { d.s } \\
--\end{array}$ \\
\hline 4 & $\begin{array}{c}0-25 \\
25-50 \\
50-90 \\
90-150 \\
\end{array}$ & $\begin{array}{l}7.5 \mathrm{YR} \\
7.5 \mathrm{YR} \\
7.5 \mathrm{YR} \\
7.5 \mathrm{YR} \\
\end{array}$ & $\begin{array}{l}5 / 8 \\
5 / 4 \\
5 / 6 \\
5 / 6 \\
\end{array}$ & $\begin{array}{l}7.5 \mathrm{YR} \\
7.5 \mathrm{YR} \\
7.5 \mathrm{YR} \\
7.5 \mathrm{YR} \\
\end{array}$ & $\begin{array}{l}4 / 6 \\
4 / 4 \\
4 / 6 \\
3 / 4 \\
\end{array}$ & $\begin{array}{l}\text { SCL } \\
\text { SCL } \\
\text { SCL } \\
\text { SCL } \\
\end{array}$ & $\begin{array}{l}\text { w.c.s.ang b } \\
\text { w.m.ang b } \\
\text { mod.f.ang } \\
\text { mod.f.ang }\end{array}$ & $\begin{array}{l}\text { st pl } \\
\text { st pl } \\
\text { st pl } \\
\text { st pl }\end{array}$ & $\begin{array}{l}\bmod \\
\bmod \\
\bmod \\
\bmod \end{array}$ & $\begin{array}{l}\text { d.s } \\
\text { d.s } \\
\text { d.s } \\
--\end{array}$ \\
\hline 6 & $\begin{array}{c}0-25 \\
25-65 \\
65-100\end{array}$ & $\begin{array}{l}10 \mathrm{YR} \\
10 \mathrm{YR} \\
10 \mathrm{YR}\end{array}$ & $\begin{array}{l}5 / 6 \\
5 / 8 \\
5 / 6\end{array}$ & $\begin{array}{l}10 \mathrm{YR} \\
10 \mathrm{YR} \\
10 \mathrm{YR}\end{array}$ & $\begin{array}{l}4 / 6 \\
4 / 6 \\
4 / 6\end{array}$ & $\begin{array}{c}\text { SC } \\
\text { SC } \\
\text { SCL }\end{array}$ & $\begin{array}{c}\text { w.c.s.ang } \\
\text { mod.m.s.ang } \\
\text { b } \\
\text { mod.m.s.ang } \\
\text { b }\end{array}$ & $\begin{array}{l}\text { st pl } \\
\text { st pl } \\
\text { st pl }\end{array}$ & $\begin{array}{l}\text { w } \\
\text { w } \\
\text { w }\end{array}$ & $\begin{array}{l}\text { d.s } \\
\text { c.s }\end{array}$ \\
\hline 8 & $\begin{array}{c}0-15 \\
15-45 \\
45-70 \\
70-100 \\
100-120\end{array}$ & $\begin{array}{l}10 \mathrm{YR} \\
10 \mathrm{YR} \\
10 \mathrm{YR} \\
10 \mathrm{YR} \\
10 \mathrm{YR}\end{array}$ & $\begin{array}{l}6 / 6 \\
6 / 4 \\
5 / 4 \\
5 / 6 \\
5 / 4\end{array}$ & \begin{tabular}{|l|}
$10 \mathrm{YR}$ \\
$10 \mathrm{YR}$ \\
$10 \mathrm{YR}$ \\
$10 \mathrm{YR}$ \\
$10 \mathrm{YR}$ \\
\end{tabular} & $\begin{array}{l}4 / 6 \\
5 / 4 \\
5 / 3 \\
5 / 4 \\
5 / 3\end{array}$ & \begin{tabular}{|c|} 
C \\
SL \\
SC \\
SCL \\
SCL
\end{tabular} & $\begin{array}{c}\text { w.c.s.ang } \\
\text { ma } \\
\text { ma } \\
\text { ma } \\
\text { ma }\end{array}$ & $\begin{array}{l}\text { st pl } \\
\text { slst slpl } \\
\text { st pl } \\
\text { st pl } \\
\text { st pl }\end{array}$ & $\begin{array}{l}\text { mod } \\
\text { mod } \\
\text { mod } \\
\text { mod } \\
\text { mod }\end{array}$ & $\begin{array}{l}\text { c.s } \\
\text { c.s } \\
\text { d.s } \\
--\end{array}$ \\
\hline 10 & $\begin{array}{c}0-15 \\
15-35 \\
35-50 \\
50-70 \\
70-100 \\
\end{array}$ & $\begin{array}{l}7.5 \mathrm{YR} \\
7.5 \mathrm{YR} \\
7.5 \mathrm{YR} \\
7.5 \mathrm{YR} \\
7.5 \mathrm{YR}\end{array}$ & $\begin{array}{l}5 / 8 \\
5 / 4 \\
5 / 6 \\
5 / 6 \\
5 / 6\end{array}$ & \begin{tabular}{|l|}
$7.5 \mathrm{YR}$ \\
$7.5 \mathrm{YR}$ \\
$7.5 \mathrm{YR}$ \\
$7.5 \mathrm{YR}$ \\
$7.5 \mathrm{YR}$ \\
\end{tabular} & $\begin{array}{l}4 / 6 \\
4 / 4 \\
4 / 6 \\
3 / 4 \\
3 / 4\end{array}$ & \begin{tabular}{|c|} 
C \\
SC \\
SCL \\
SL \\
SL \\
\end{tabular} & \begin{tabular}{|c|} 
w.c.s.ang b \\
w.m.ang b \\
mod.f.ang \\
ma. \\
Ma.
\end{tabular} & $\begin{array}{c}\text { st pl } \\
\text { st pll } \\
\text { st pl } \\
\text { so.sl.st. } \\
\text { so.sl.st. }\end{array}$ & $\begin{array}{l}\bmod \\
\bmod \\
\bmod \\
\bmod \end{array}$ & $\begin{array}{l}\text { d.s } \\
\text { d.s } \\
\text { d.s } \\
--\end{array}$ \\
\hline
\end{tabular}

Soil Taxonomy

Based on the morphological, physical and chemical characteristics of the studied soil profiles as well as meteorological data, the studied soils were 
classified up to the family level according to the American System of Soil Taxonomy (USDA, 2010).

\section{Land evaluation}

The soil under investigation were evaluated using the two systems namely, land capability classification of Sys et al. (1991) and soil suitability classification of certain crops based on the concepts outlined by Sys et al. (1993). The main soil parameters used in this system are climate, soil depth, texture, gravel content, $\mathrm{CaCO}_{3}$ percent, gypsum percent, salinity (ECe), alkalinity (SAR), slope pattern and different conditions. A suitability index of 12 crops for the studied soils was done according to this system.

\section{Crop water requirement}

The crop water requirements were calculated using crop wat. program. The program determines ETo using Penmon - Monteith method, (Allen, 1998). The climatic data of El- Dakhla Oases, Climatological Normals for Egypt (2010) and Central Laboratory for Agriculture of Climate (CLAC 2010) were used.

\section{Results and Discussion}

El- Dakhla Oases soils consists mainly of five dominant physiographic units. These are, Playa, Sabkha, Cultivated plain, Sand dunes, Sand sheets and Peniplain. A breif notes about the identified physiographic units were shown in Table 2. Soil characteristics of the physiograohic units could be discussed and classified according to USDA (2010) based on the data in Tables 3, 4 and 5. Some of these characteristics could be summarized in the following lines:-

TABLE 3. Particle Size Distribution, Texture class, $\mathrm{CaCO}_{3}$ Content and $\mathrm{OM} \%$ of the Studied Soil Profiles.

\begin{tabular}{|c|c|c|c|c|c|c|c|c|c|}
\hline \multirow{2}{*}{$\begin{array}{c}\text { Prof. } \\
\text { No. }\end{array}$} & \multirow{2}{*}{$\begin{array}{c}\text { Depth } \\
\text { Cm }\end{array}$} & \multirow{2}{*}{$\begin{array}{c}\text { gravels } \\
\%\end{array}$} & \multicolumn{4}{|c|}{ Particale size distribution $\%$} & \multirow{2}{*}{ 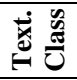 } & \multirow{2}{*}{$\begin{array}{c}\mathrm{CaCO}_{3} \\
\%\end{array}$} & \multirow{2}{*}{$\begin{array}{c}\text { OM } \\
\%\end{array}$} \\
\hline & & & C.S & F.S & Silt & clay & & & \\
\hline \multicolumn{10}{|c|}{ Playa } \\
\hline \multirow{4}{*}{1} & $0-30$ & 37 & 38.15 & 25.22 & 8.12 & 28.51 & SCL & 2.83 & 0.003 \\
\hline & $30-60$ & 37 & 55.68 & 20.79 & 2.30 & 21.23 & SCL & 1.89 & 0.41 \\
\hline & $60-80$ & 36 & 71.62 & 14.37 & 0.13 & 13.88 & LS & 1.62 & 0.003 \\
\hline & $80-150$ & 39 & 66.40 & 15.36 & 2.73 & 15.51 & SL & 135 & 0.41 \\
\hline \multirow{2}{*}{2} & $0-10$ & 24 & 40.81 & 17.59 & 6.07 & 30.53 & SCL & 2.97 & 0.39 \\
\hline & $10-50$ & 38 & 56.78 & 32.61 & 0.46 & 10.15 & LS & 3.10 & 0.003 \\
\hline \multicolumn{10}{|c|}{ Sabkha } \\
\hline \multirow{2}{*}{5} & $0-10$ & 27 & 37.68 & 21.47 & 10.47 & 30.38 & SCL & 4.19 & 0.02 \\
\hline & $10-40$ & 39 & 82.17 & 15.04 & 1.66 & 1.13 & $\mathrm{~S}$ & 3.92 & 1.05 \\
\hline \multicolumn{10}{|c|}{ Sand Sheets } \\
\hline \multirow{4}{*}{7} & $0-15$ & 27 & 68.46 & 27.93 & 1.37 & 2.24 & $\mathrm{~S}$ & 13.50 & 0.28 \\
\hline & $15-55$ & 25 & 78.76 & 12.97 & 0.43 & 7.84 & LS & 10.13 & 1.48 \\
\hline & $55-85$ & 36 & 87.60 & 11.79 & 0.11 & 3.41 & $\mathrm{~S}$ & 12.96 & 0.07 \\
\hline & $85-110$ & 37 & 93.68 & 4.68 & 0.45 & 1.19 & $\mathrm{~S}$ & 11.50 & 0.39 \\
\hline \multicolumn{10}{|c|}{ Peniplain } \\
\hline \multirow{4}{*}{9} & $0-15$ & 20 & 32.77 & 25.70 & 2.21 & 39.92 & $\mathrm{SC}$ & 6.48 & 0.003 \\
\hline & $15-55$ & 24 & 19.48 & 17.12 & 7.01 & 56.20 & $\mathrm{C}$ & 0.81 & 0.69 \\
\hline & $55-75$ & 39 & 23.35 & 27.72 & 0.92 & 48.01 & $\mathrm{SC}$ & 1.62 & 0.14 \\
\hline & $75-110$ & 39 & 33.33 & 13.37 & 0.92 & 52.38 & $\mathrm{SC}$ & 0.95 & 0.34 \\
\hline
\end{tabular}

Egypt. J. Soil Sci. 56, No. 1 (2016) 
TABLE 3. Cont.

\begin{tabular}{|c|c|c|c|c|c|c|c|c|c|}
\hline \multirow{2}{*}{$\begin{array}{l}\text { Prof. } \\
\text { No. }\end{array}$} & \multirow{2}{*}{$\begin{array}{c}\text { Depth } \\
\text { Cm }\end{array}$} & \multirow{2}{*}{$\begin{array}{c}\text { gravels } \\
\%\end{array}$} & \multicolumn{4}{|c|}{ Particle size distribution \% } & \multirow{2}{*}{$\begin{array}{l}\text { Text. } \\
\text { Class }\end{array}$} & \multirow{2}{*}{$\begin{array}{c}\mathrm{CaCO}_{3} \\
\%\end{array}$} & \multirow{2}{*}{$\underset{\%}{\mathrm{OM}}$} \\
\hline & & & C.S & F.S & Silt & Clay & & & \\
\hline \multicolumn{10}{|c|}{ (Plain) Cultivated area } \\
\hline \multirow{4}{*}{3} & $0-10$ & 40 & 30.94 & 39.57 & 3.47 & 26.03 & SCL & 5.67 & 0.48 \\
\hline & $10-35$ & 4 & 32.45 & 24.43 & 12.49 & 30.63 & SCL & 7.02 & 0.48 \\
\hline & $35-65$ & 7 & 46.0 & 23.15 & 2.91 & 27.94 & SCL & 6.34 & 0.28 \\
\hline & $65-100$ & 20 & 47.79 & 21.33 & 8.48 & 22.40 & SCL & 5.40 & 0.28 \\
\hline \multirow{4}{*}{4} & $0-25$ & 19 & 30.56 & 44.03 & 0.52 & 24.89 & SCL & 6.75 & 0.21 \\
\hline & $25-50$ & 18 & 18.88 & 45.91 & 2.99 & 32.22 & SCL & 6.21 & 0.21 \\
\hline & $50-90$ & 19 & 45.97 & 27.31 & 1.09 & 25.63 & SCL & 6.21 & 0.34 \\
\hline & $90-150$ & 15 & 49.68 & 19.47 & 1.77 & 29.08 & SCL & 5.94 & 0.34 \\
\hline \multirow{3}{*}{6} & $0-25$ & 36 & 13.44 & 43.76 & 1.93 & 40.87 & $\mathrm{SC}$ & 3.78 & 0.41 \\
\hline & $25-65$ & 27 & 12.85 & 45.30 & 1.42 & 40.43 & $\mathrm{SC}$ & 1.50 & 0.34 \\
\hline & $65-100$ & 39 & 35.51 & 36.73 & 1.51 & 26.25 & SCL & 3.78 & 0.69 \\
\hline \multirow{5}{*}{8} & $0-15$ & 39 & 11.41 & 22.54 & 6.75 & 59.30 & $\mathrm{C}$ & 11.07 & 0.69 \\
\hline & $15-45$ & 37 & 16.27 & 32.36 & 49.47 & 1.90 & SL & 5.13 & 0.14 \\
\hline & $45-70$ & 25 & 22.06 & 34.7 & 1.65 & 41.59 & $\mathrm{SC}$ & 4.59 & 0.48 \\
\hline & $70-100$ & 27 & 17.41 & 47.04 & 1.35 & 34.21 & SCL & 5.40 & 0.14 \\
\hline & $100-120$ & 29 & 29.14 & 47.34 & 2.65 & 20.87 & SCL & 4.32 & 0.21 \\
\hline \multirow{5}{*}{10} & $0-15$ & 12 & 4.74 & 23.44 & 15.72 & 56.10 & $\mathrm{C}$ & 4.32 & 0,69 \\
\hline & $15-35$ & 9 & 11.42 & 39.27 & 15.31 & 44.00 & $\mathrm{SC}$ & 2.56 & 0.14 \\
\hline & $35-50$ & 29 & 21.16 & 44.62 & 2.30 & 31.92 & SCL & 1.75 & 0.003 \\
\hline & $50-70$ & 9 & 28.74 & 50.43 & 1.95 & 18.88 & SL & 1.35 & 0.003 \\
\hline & $70-100$ & 15 & 25.82 & 54.24 & 3.98 & 15.96 & SL & 2.30 & 0.50 \\
\hline
\end{tabular}

TABLE 4. Some chemical analyses of the studied soil profiles.

\begin{tabular}{|c|c|c|c|c|c|c|c|c|c|c|c|c|c|}
\hline \multirow[b]{2}{*}{ 连 } & \multirow[b]{2}{*}{ 营 } & \multirow[b]{2}{*}{ pH } & \multirow[b]{2}{*}{$\begin{array}{c}\mathrm{ECe} \\
(\mathrm{dS} / \mathrm{m})\end{array}$} & \multicolumn{4}{|c|}{ Anions } & \multicolumn{4}{|c|}{ Cations } & \multirow[b]{2}{*}{$\underset{\%}{\text { Gyp. }}$} & \multirow[b]{2}{*}{ SAR } \\
\hline & & & & "ृ & $\dot{e}^{m}$ & $\dot{0}$ & $\ddot{ம}^{+}$ & تَ & $\stackrel{+}{+\infty}^{+}$ & ${ }^{+} \boldsymbol{\pi}$ & $\stackrel{+}{\longleftarrow}$ & & \\
\hline \multicolumn{14}{|c|}{ Playa } \\
\hline \multirow{4}{*}{1} & $0-30$ & 7.78 & 61.13 & - & 7.69 & 2150. & 377.4 & 641.02 & 1334.28 & 491.97 & 67.83 & 0.12 & 15.65 \\
\hline & $30-60$ & 8.20 & 27.39 & - & 3.08 & 220.0 & 178.5 & 46.15 & 32.86 & 317.4 & 5.18 & 1.42 & 50.54 \\
\hline & 60-80 & 8.22 & 36.37 & - & 4.90 & 360.0 & 83.63 & 51.28 & 17.86 & 371.36 & 8.03 & 1.90 & 63.16 \\
\hline & $80-150$ & 8.29 & 19.04 & - & 3.69 & 88.0 & 160.9 & 46.28 & 37.8 & 163.46 & 5.18 & 1.09 & 25.23 \\
\hline \multirow{2}{*}{2} & $0-10$ & 7.64 & 23.22 & - & 2.46 & 301.0 & 42.05 & 135.89 & 22.13 & 184.1 & 3.39 & 0.82 & 20.71 \\
\hline & $10-50$ & 7.53 & 28.48 & - & 3.69 & 440.0 & 8.70 & 151.28 & 46.25 & 250.75 & 4.11 & 1.20 & 12.15 \\
\hline \multicolumn{14}{|c|}{ Sabkha } \\
\hline \multirow{2}{*}{5} & $0-10$ & 6.95 & 14.72 & - & 6.15 & 130.0 & 48.67 & 56.41 & 5.34 & 120.39 & 2.68 & 15.99 & 21.69 \\
\hline & $10-40$ & 7.44 & 54.42 & - & 12.65 & 2930. & 36049.9 & 256.41 & 317.66 & 38400.0 & 18.21 & 4.11 & 2266.8 \\
\hline \multicolumn{14}{|c|}{ Sand Sheets } \\
\hline \multirow{4}{*}{7} & $0-15$ & 7.51 & 15.83 & - & 1.54 & 390.0 & 62.06 & 201.28 & 1.22 & 236.46 & 14.64 & 1.16 & 23.50 \\
\hline & $15-55$ & 7.54 & 32.92 & - & 2.66 & 420.0 & 281.8 & 394.87 & 91.55 & 803.31 & 11.78 & 0.90 & 13.03 \\
\hline & $55-85$ & 7.65 & 24.49 & - & 2.46 & 308.0 & 170.8 & 256.41 & 67.04 & 149.18 & 8.21 & 0.40 & 11.73 \\
\hline & $85-110$ & 7.63 & 16.88 & & 3.08 & 156.0 & 136.7 & 115.38 & 82.15 & 93.63 & 4.64 & 0.34 & 9.42 \\
\hline \multicolumn{14}{|c|}{ Peniplain } \\
\hline \multirow{4}{*}{9} & $0-15$ & 7.87 & 10.74 & - & 1.85 & 68.0 & 85.57 & 51.28 & 15.38 & 82.52 & 6.24 & 4.34 & 14.30 \\
\hline & $15-55$ & 8.06 & 39.27 & - & 2.32 & 240.0 & 265.6 & 56.41 & 27.54 & 412.62 & 11.42 & 2.19 & 63.67 \\
\hline & $55-75$ & 8.21 & 45.44 & - & 9.22 & 250.0 & 628.2 & 123.20 & 19.95 & 730.02 & 9.28 & 0.89 & \begin{tabular}{|l}
84.78 \\
\end{tabular} \\
\hline & $75-110$ & 8.22 & 28.21 & - & 4.32 & 176.0 & 276.1 & 66.66 & 61.69 & 323.75 & 4.28 & 3.98 & 40.42 \\
\hline
\end{tabular}


TABLE 4. Cont.

\begin{tabular}{|c|c|c|c|c|c|c|c|c|c|c|c|c|c|}
\hline \multirow{2}{*}{ 总 } & \multirow{2}{*}{ 竞 } & \multirow[b]{2}{*}{ a } & \multirow{2}{*}{ 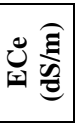 } & \multicolumn{4}{|c|}{ Anions } & \multicolumn{4}{|c|}{ Cations } & \multirow[b]{2}{*}{$\underset{\%}{\text { Gyp. }}$} & \multirow[b]{2}{*}{ SAR } \\
\hline & & & & "ृ & $e_{0}^{m}$ & ப் & 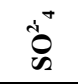 & Uં & $\sum^{+50}$ & ${ }^{+}$ & $\stackrel{+}{*}$ & & \\
\hline \multicolumn{14}{|c|}{ (Plain) Cultivated area } \\
\hline \multirow{4}{*}{3} & $0-10$ & 7.63 & 4.86 & - & 9.23 & 18.0 & 37.11 & 30.76 & 6.28 & 22.75 & 4.55 & 0.10 & 5.29 \\
\hline & $10-35$ & 7.44 & 9.29 & - & 8.46 & 59.0 & 39.13 & 45.51 & 29.8 & 24.50 & 6.78 & 0.35 & 3.99 \\
\hline & $35-65$ & 7.53 & 3.04 & & 6.46 & 15.0 & 21.26 & 19.23 & 6.70 & 11.43 & 5.36 & 0.09 & 3.18 \\
\hline & $65-100$ & 7.56 & 2.58 & & 3.38 & 3.0 & 11.85 & 8.97 & 3.90 & 4.13 & 1.23 & 0.14 & 1.63 \\
\hline \multirow{4}{*}{4} & $0-25$ & 7.71 & 2.82 & & 4.61 & 16.0 & 6.49 & 10.89 & 1.45 & 13.65 & 1.11 & 0.14 & 5.50 \\
\hline & $25-50$ & 7.96 & 1.44 & - & 3.08 & 8.74 & 12.82 & 1.37 & 4.76 & 0.87 & 19.82 & 0.17 & 0.50 \\
\hline & $50-90$ & 8.30 & 4.34 & - & 3.08 & 14.0 & 43.8 & 44.87 & 8.21 & 10.16 & 1.64 & 0.34 & 1.97 \\
\hline & $90-150$ & 7.53 & 4.64 & 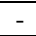 & 5.69 & 16.08 & 54.0 & 43.58 & 8.27 & 14.91 & 9.01 & 1.45 & 2.93 \\
\hline \multirow{3}{*}{6} & $0-25$ & 7.80 & 1.53 & - & 4.61 & 7.0 & 12.73 & 11.54 & 0.82 & 4.11 & 0.87 & 0.18 & 4.48 \\
\hline & $25-65$ & 7.81 & 0.93 & - & 3.99 & 3.0 & 3.03 & 5.13 & 2.28 & 2.16 & 0.45 & 0.13 & 1.125 \\
\hline & $65-100$ & 8.03 & 0.68 & - & 2.30 & 3.0 & 6.52 & 5.13 & 2.28 & 3.86 & 0.55 & 0.23 & 2.00 \\
\hline \multirow{4}{*}{8} & \begin{tabular}{|l|l|}
$0-15$ \\
\end{tabular} & 7.95 & 3.68 & - & 1.69 & 12.0 & 73.25 & 37.18 & 6.03 & 41.23 & 2.5 .0 & 3.33 & 8.87 \\
\hline & $15-45$ & 8.08 & 37.09 & - & 4.32 & 102.1 & 360.0 & 51.25 & 7.98 & 390.4 & 16.78 & 3.47 & 71.76 \\
\hline & $45-100$ & 7.98 & 22.22 & - & 4.32 & 160.0 & 187.4 & 82.05 & 41.40 & 222.8 & 6.07 & 2.43 & 28.27 \\
\hline & \begin{tabular}{|l|}
$100-$ \\
120
\end{tabular} & 8.06 & 17.68 & - & 3.99 & 90.0 & 110.6 & 49.38 & 4.94 & 144.42 & 5.89 & 1.59 & 27.72 \\
\hline \multirow{5}{*}{10} & $0-15$ & 7.97 & 5.17 & 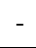 & 2.31 & 14.38 & 53.0 & 338.46 & 1.04 & 26.98 & 3.21 & 1.47 & 60.8 \\
\hline & 15-35 & 7.87 & 30.1 & - & 9.23 & 320.0 & 34.68 & 21.79 & 72.03 & 260.27 & 9.82 & 1.29 & 37.99 \\
\hline & $35-50$ & 7.85 & 26.0 & - & 6.10 & 236.0 & 88.03 & 92.30 & 6.46 & 225.35 & 6.07 & 1.38 & 32.06 \\
\hline & \begin{tabular}{|l|}
$50--70$ \\
\end{tabular} & 7.94 & 20.0 & - & 3.07 & 164.0 & 96.43 & 130.76 & 29.73 & 99.98 & 3.03 & 0.46 & 11.15 \\
\hline & $70-100$ & 7.83 & 8.52 & - & 1.84 & 64.0 & 35.03 & 38.46 & 32.26 & 38.08 & 1.07 & 0.14 & 6.86 \\
\hline
\end{tabular}

TABLE 5. Soil classification categories of the studied profiles (according to USDA 2010).

\begin{tabular}{|c|c|c|c|c|c|}
\hline Order & $\begin{array}{c}\text { Subor } \\
\text { der }\end{array}$ & $\begin{array}{l}\text { Great } \\
\text { group }\end{array}$ & $\begin{array}{c}\text { Sub great } \\
\text { group }\end{array}$ & Soil Families & $\begin{array}{l}\text { Profile } \\
\text { No. }\end{array}$ \\
\hline \multirow{7}{*}{ Aridisols } & \multirow{7}{*}{ Salids } & \multirow{7}{*}{ Haplosalids } & $\begin{array}{l}\text { Calcic } \\
\text { Haplosalids }\end{array}$ & $\begin{array}{l}\text { Sandy skeletal, siliceous, } \\
\text { hyperthermic, deep }\end{array}$ & 7 \\
\hline & & & $\begin{array}{l}\text { Duric } \\
\text { Haplosalids }\end{array}$ & $\begin{array}{l}\text { Sandy skeletal, mixed, } \\
\text { hyperthermic, shallow }\end{array}$ & 2 \\
\hline & & & \multirow{4}{*}{$\begin{array}{l}\text { Typic } \\
\text { Haplosalids }\end{array}$} & $\begin{array}{l}\text { Fine loamy skeletal over Sandy } \\
\text { skeletal, mixed, hyperthermic, deep }\end{array}$ & 1 \\
\hline & & & & $\begin{array}{l}\text { Clayey skeletal, mixed, } \\
\text { hyperthermic, deep }\end{array}$ & 9 \\
\hline & & & & $\begin{array}{l}\text { Coarse loamy skeletal over clayey, } \\
\text { mixed, hyperthermic, deep }\end{array}$ & 8 \\
\hline & & & & $\begin{array}{l}\text { Fine loamy over Coarse loamy, mixed, } \\
\text { hyperthermic, moderately deep }\end{array}$ & 10 \\
\hline & & & $\begin{array}{l}\text { Lithic } \\
\text { Gypsisalids }\end{array}$ & $\begin{array}{l}\text { Sandy skeletal, siliceous, } \\
\text { hyperthermic, shallow, lithic }\end{array}$ & 5 \\
\hline \multirow{3}{*}{ Entisols } & Fluvents & Torrifluvents & $\begin{array}{l}\text { Typic } \\
\text { Torrifluvents }\end{array}$ & $\begin{array}{l}\text { Clayey over fine loamy skeletal, } \\
\text { mixed, hyperthermic, moderately deep }\end{array}$ & 6 \\
\hline & \multirow[b]{2}{*}{ Orthents } & \multirow[b]{2}{*}{ Torriorthents } & \multirow{2}{*}{$\begin{array}{l}\text { Typic } \\
\text { Torriorthents }\end{array}$} & \multirow{2}{*}{$\begin{array}{l}\text { Fine loamy, mixed, hyperthermic, } \\
\text { deep }\end{array}$} & 3 \\
\hline & & & & & 4 \\
\hline
\end{tabular}

Egypt. J. Soil Sci. 56, No. 1 (2016) 


\section{1- Soils of playa}

This physiographic units represented by profiles No. 1 and 2 cover an area of about $84.5 \mathrm{~km}^{2}$ (20280 feddens). The analytical data show that soil texture class varied from loamy sand to sandy clay loam. $\mathrm{CaCO}_{3}$ content is very low and ranged from 1.35 to $3.10 \%$. Gypsum content is generally less than $1.9 \%$. Organic matter content is extremely low, not exceeding $0.41 \%$. Soil $\mathrm{pH}$ values ranged between 7.53 and 8.29 indicating that these soils are slightly to moderately alkaline. Data presented in Table 4 revealed that the studied playa soils are highly to extremely saline and characterized by different salinity levels from 19.04 to $61.13 \mathrm{sm}^{-1}$. Sodium ions are the predominate soluble cations followed by $\mathrm{Ca}^{++}$and $\mathrm{Mg}^{++}$, while $\mathrm{K}^{+}$is rather low, except for the surface layer of profile No. 1 . where $\mathrm{Mg}^{++}$exceeds $\mathrm{Na}^{+}$. Soluble anions are dominated by $\mathrm{Cl}^{-}$followed $\mathrm{SO}_{4}{ }^{\circ}$ and then $\mathrm{HCO}_{3}{ }^{-}$. SAR varied from 12.15 to 63.16 , indicating that these soils are sodic. The soils of this physiographic unit are classified as :

1- Typic Haplosalids, fine loamy over sand skeletal, mixed, hyperthermic (profile 1)

2- Duric Haplosalids, sand skeletal, mixed, hyperthermic (profile 2).

\section{2- Soils of sabkha}

This physiographic unit is represented by profile No.5. and covers an area of about $12.34 \mathrm{~km}^{2}$ (2962 feddans). Data in Table 3 show that the depth of these soils is around $40 \mathrm{~cm}$. It is limited by a lithic contact. Soil texture class is sandy clay loam in the surface layer changed into sand in the $10-40 \mathrm{~cm}$ depth. Calcium carbonate content is very low and varied within narrow limit (3.92-4.19\%), while organic matter content is extremely low, not exceeding $1.05 \%$, soil reaction ranges from 6.95 to 7.44 (neutral to slightly alkaline). Electrical conductivity (ECe) ranges from 14.72 to $54.42 \mathrm{dSm}^{-1}$ indicating that these soils are moderately saline in the surface layer and extremely saline in the deepest layer. Soluble cations follow the order $\mathrm{Na}^{+}>\mathrm{Ca}^{++}>\mathrm{Mg}^{++}>\mathrm{K}^{+}$, while soluble anions follow the order $\mathrm{SO}_{4}{ }^{\circ}>\mathrm{Cl}^{-}>\mathrm{HCO}^{-}$. Gypsum content varied from 4.11 to $15.99 \%$ and tends to decrease within the depth. The soils of this physiographic unit are sodic soils, where SAR values ranged from 21.69 to 2266.8 . Soils of this unit are classified as Lithic Gypsisalids, sandy skeletal, siliceous, hyperthermic, shallow (profile 5).

\section{3- Soils of sand sheets}

This unit is represented by soil profile No.7 and occupied about $188.8 \mathrm{~km}^{2}$ (45312 feddans). Data show that the soils of this unit are more than $100 \mathrm{~cm}$ depth (deep). The analytical data reveal that soil texture class is sand or loamy sand in the different layers of the representative soil profile. $\mathrm{CaCO}_{3}$ content ranges between 10.13 and $13.5 \%$ with an irregular distribution pattern within the depth. Organic matter is extremely low and varied from 0.07 to $1.48 \%$. Soil reaction is slightly alkaline ( $\mathrm{pH}$ values are 7.51-7.65). Soluble salts content ranged between 15.83 and $32.92 \mathrm{dSm}^{-1}$ showing that these soils are moderately to extremely saline. Soluble cations are dominated by $\mathrm{Na}^{+}$and / or $\mathrm{Ca}^{++}$followed by $\mathrm{Mg}^{++}$, while soluble $\mathrm{K}^{+}$is the least abundant. On the other hand, soluble 
anions are dominated by $\mathrm{Cl}^{-}$followed by $\mathrm{SO}_{4}{ }^{=}$and $\mathrm{HCO}_{3}{ }^{-}$. Gypsum content is very low, not exceeding $1.16 \%$. SAR values ranged from 9.42 to 23.5 .

Soil characteristics of the second horizon meet the requirement of both salic and calcic horizons. Soils of this unit are classified as Calcic Haplosalids, sandy skeletal, mixed, hyperthermic, deep. (profile 7).

\section{Soils of peniplain}

This physiographic unit is represented by profile No. 9. Its area occupied about $156.4 \mathrm{~km}^{2}$ (37536 feddans). The obtained data show that the soils are deep $(>100 \mathrm{~cm})$. The soil texture class is sandy clay in the surface and deepest layers, mean while it is clay in the subsurface layer. $\mathrm{CaCO}_{3}$ content varies from 0.81 to $6.48 \%$. The distribution pattern of $\mathrm{CaCO}_{3}$ content does not portray any specific pattern within the depth. Organic matter content is very low, not exceeding $0.69 \%$. Soil reaction is between 7.87 and 8.22 showing that these soils are strongly alkaline. Soluble salts content vary between 10.74 and $45.44 \mathrm{dSm}^{-1}$ (moderately to extremely saline). Sodium is the dominant soluble cation followed by $\mathrm{Ca}^{++}, \mathrm{Mg}^{++}$and $\mathrm{K}^{+}$. SO4${ }^{=}$is the dominant soluble anion followed by $\mathrm{Cl}^{-}$and $\mathrm{HCO}_{3}^{-}$. Gypsum content ranged from 0.89 to $4.34 \%$ with an irregular distribution pattern within the depth. SAR values are more than 13 indicating that the soils of peniplain are sodic soils. These soils have a salic horizon in the soil depth from 25-100 cm (Control section). Soils of this unit are classified as: Typic Haplosalids, clayey skeletal, hyperthermic (profile 9).

\section{Soils of cultivated plain}

This unit is represented by profiles No. 3, 4, 6.8 and 10 and covers an area about $933.3 \mathrm{~km}^{2}$ (223992 feddans). The obtained results reveal that depth of these soils is between 95 and $150 \mathrm{~cm}$. Soil texture varied from sandy loam to clay. Both representative profiles (No. 3 and 4) have the same pattern of sedimentation in all profile layers, where texture class is sandy clay loam. Calcium carbonate content ranges from 1.35 to $11.07 \%$ with an irregular distribution pattern within soil profile depth. Organic matter content is very low, $0.69 \%$. The soil reaction ranges from 7.44 to 8.30 indicating that these soils are slightly to moderately alkaline, soluble salts content varies from 0.68 to 37.09 dSm-1 (non to extremely saline). Soluble cations are dominated with $\mathrm{Ca}^{++}$and / or $\mathrm{Na}^{+}$followed by $\mathrm{Mg}^{++}$and $\mathrm{K}^{+}$, while soluble anions are dominated by $\mathrm{SO}_{4}{ }^{=}$ and $\mathrm{Cl}^{-}$followed by $\mathrm{HCO}_{3}{ }^{-}$. Gypsum content varies from 0.09 to $3.47 \%$, SAR values ranged between 1.63 and 71.76 indicating that these soils are non sodic to strongly sodic soils. The soils of this unit are classified as:

1- Typic Torriorthents, fine loamy, mixed, hyperthermic (profiles 3 and 4)

2- Typic Torriorthents, clayey over fine loamy skeletal, mixed, hyperthermic (profile 6).

3- Typic Haplosalids, coarse loamy skeletal over clay, mixed, hyperthermic, (profile 8).

4- Typic Haplosalids, fine loamy over coarse loamy, mixed, hyperthermic (profile10). 


\section{Statistical size parameters}

Statistical measures (Folk and Ward, 1957) serve a guide in the explanation of the environment of deposition and agents of transportation. Data in Table 6 reveal that, the soils of playa, represented by profiles 1 and 2 have sorting values that ranged between 1.1 and $1.7 \mathrm{Q}$, indicating that the sediments are poorly sorted throughout the entire profile depths. This indictates that their sediments are transported and deposited under water action. Values of skewness indicate that all layers of the representative profiles are strong fine skewed and near symmetry in the top layer of profile 1.

These soils have a tail towards fineness. The kurtosis values ranged from 0.45 and $1.35 \mathrm{Q}$, indicating that the sediments are meso kurtic, extremely lepto kurtic and platy kurtic. This leads to the suggestion that the soils are mainly formed under water action.

With regard to the soils of sabkha (profile 5), these soils constitute poorly sorted sediments in the surface layer and moderately sorted in the subsurface layer, this indicates that the surface layer is transported and deposited by water action, while the subsurface layer is transported and deposited under combined action of both water and wind. The sediments constituting profile 5 is fine and very fine skewed in the surface and subsurface layers, respectively. Graphic kurtosis indicates that the sediments constituting profile 5 is meso kurtic in the top layer and extremely leptokurtic in the subsurface layers.

Sorting values of the sand sheets showed that the sediments constituting profile 7 are well sorted in the top and deepest layers, sandwish a pair of poorly and moderately sorted in the middle layers. The well sorted sediments suggest that the surface and deepest layers are mainly transported and deposited by wind action, while poorly and moderately sorted sediments are transported and deposited by water or water and wind actions. Graphic skewness values in the sandy soils are coarse skewed in the surface layer and fine skewed in the subsurface and deepest layers, kurtosis of these sediments is extremely leptokurtic throughout the entire profile depths.

The obtained results of the peniplain soils (profile 9), show that the sorting values varied from 1.4 to $1.71 \propto$ indicating that the sediments of the studied soil profiles are poorly sorted sediments throughout the entire depth. This leads to suggestion that the sediments of these soils are transported and deposited by water action or weathered in situ. Values of skewness indicate that the uppermost surface layer is fine and strong fine skewed, while the deepest layer are coarse and strong coarse skewed. The kurtosis values indicate that the sediments of profile 9 are meso kurtic in the surface layer and leptok urtic in the deepest layers. 
SOLIMAN, M.M.

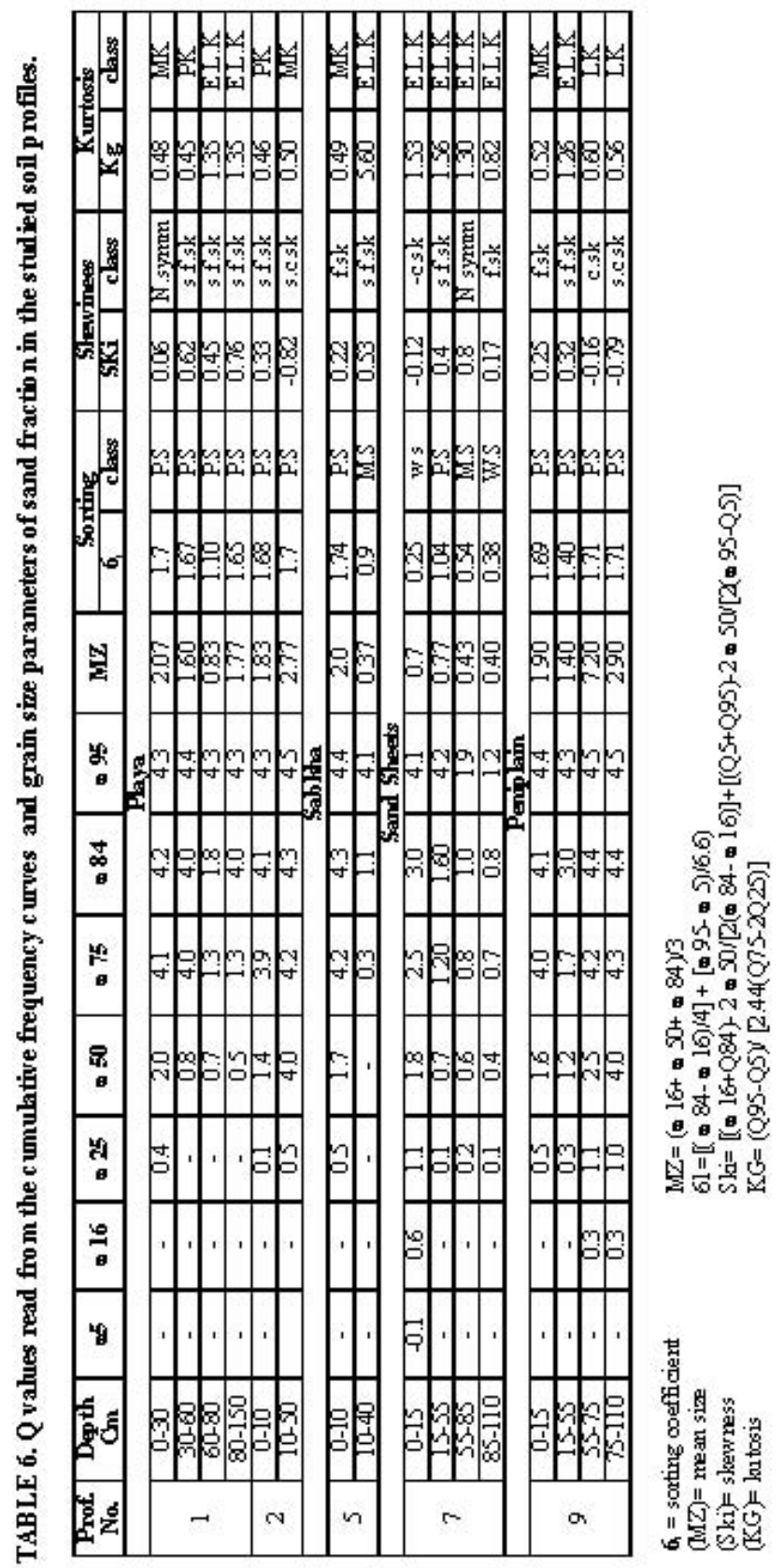

Egypt. J. Soil Sci. 56, No. 1 (2016) 

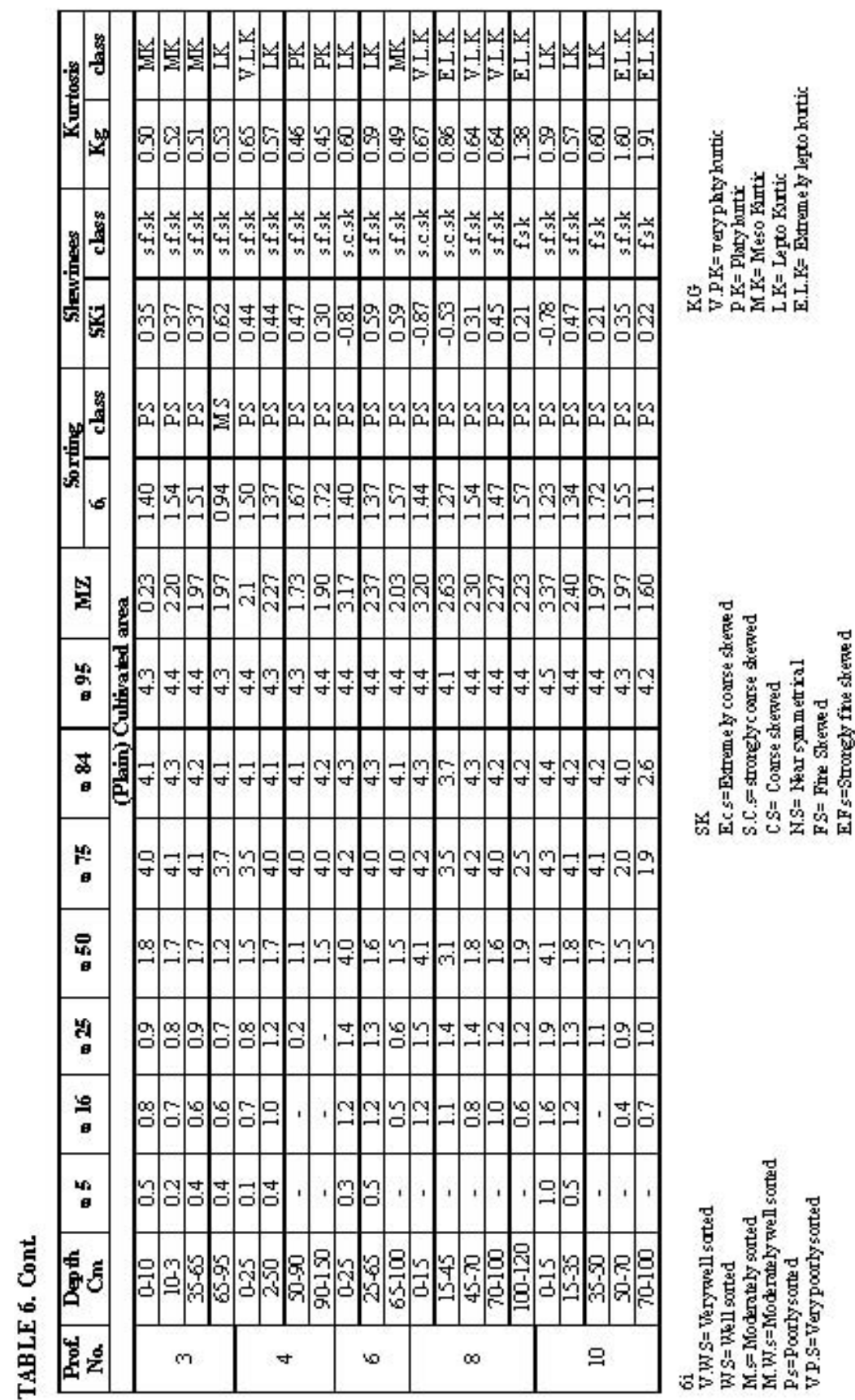

Egypt. J. Soil Sci. 56, No.1 (2016) 
Regarding the cultivated plain soils which are represented by profiles $3,4,6$, 8 and 10, data in Table 6 reveal that the standard deviation (sorting) values are in the range of 0.94 to $1.72 \propto$, the distribution of sorting of these values is almost bimodal representing the poorly sorted sediment, except for the deepest layer of profile 3, which is moderately sorted. The poorly sorted nature of sediments suggests that the soils are mainly transported and deposited by water action. Data in Table 6 reveal that skewness values are widely different and ranged from 0.87 to 0.62 \& representing bimodal distribution of skewness values indicating mixing of two modal fractions, i.e., fine and coarse sand in this case. The kurtosis $(\mathrm{KG})$ is distributed between values of 0.45 and $1.91 \propto$ representing lepto and very leptokurtic, meso kurtic and platy kurtic. The somewhat normal distribution of $(\mathrm{KG})$ values corresponds to very low-energy environment and very high modification of grain size. In conclusion, it is clear that the sediments forming the studied soils are mostly deposited under aqueous or both water and wind actions. Furthermore, the available data of the statistical size parameters reveal that the studied soil profiles are formed of non- uniform parent materials. However, the stratified condition observed in these profiles is mostly attributed to depositional variations and / or to depositional regime.

\section{Land suitability for irrigated agriculture}

a) Current land suitability

By matching between the present land properties and their rating outlined by Sys et al. (1991), the current suitability of the studied area was estimated. This aims to provide a method for suitability evaluation of irrigation water based on the standard physical and chemical chcrateristies of soil properties and their symbols used as follows : Topography $(\mathrm{t})$, wetness $(\mathrm{w})$, soil texture $\left(\mathrm{S}_{1}\right)$, soil depth $\left(\mathrm{S}_{2}\right), \mathrm{CaCO}_{3}\left(\mathrm{~S}_{3}\right)$, gypsum $\left(\mathrm{S}_{4}\right)$ and salinity and sodicity $(\mathrm{n})$.

The irrigation suitability index $(\mathrm{Ci})$ is calculated as :

$$
\mathrm{Ci}=t \times \frac{w}{100} \times \frac{S 1}{100} \times \frac{\mathrm{S} 2}{100} \times \frac{\mathrm{S} 3}{100} \times \frac{\mathrm{S} 4}{100} \frac{\mathrm{n}}{100}
$$

the order $\mathrm{S}$ : suitable for irrigation ( $\mathrm{Ci}$ is more than 25$)$.

classes $\mathrm{S}_{1}$ : $\mathrm{Ci}$ is more than 75

$\mathrm{S}_{2}: \mathrm{Ci}$ is between 50 and 75

$\mathrm{S}_{3}$; $\mathrm{Ci}$ is between 25 and 50

Order $\mathrm{N}$ : suitable for irrigation ( $\mathrm{Ci}$ is leas than 25 )

classes $\mathrm{N}_{1}$ : with limitations which can be corrected

$\mathrm{N}_{2}$ : with limitations which cannot be corrected.

Capability index for the studied soil profiles are presented in Table 7 and Fig. 2. The obtained results reveal that estimated current land suitability sub classes are given as follows:

Egypt. J. Soil Sci. 56, No. 1 (2016) 
Soils of grade $\left(S_{2}\right)$ (moderately suitable)

The soils of this grade are represented by three soil profiles (3, 4 and 6) which belong to cultivated plain soils. Suitability index $(\mathrm{Ci})$ values ranged from 56.97 to 72.68 . These values indicate a moderately suitable class. the soils have a moderate intensity of texture.

\section{Soils of grade $\left(S_{3}\right)$ (marginally suitable)}

This subclass represents the soils of playa (profile 1), peidmont soils (profile 9) and cultivated plain soils (profiles 8 and 10). Suitability index values (Ci) varied from 25.97 to 43.5 . These soils are affected by moderate intensity of texture class, and moderate to severe intensity of salinity and alkalinity.

Soils of non suitable $\left(N_{1}\right)$

The soils of this grade are represented by profiles 2 (playa), 5 (sabkha) and 7 (sand sheets). These soils have suitability index values less than 25 and affected by severe to very severe texture classes; moderate to severe salinity and alkalinity, soil depth and calcium carbonate contents.

As a general, three different limitations are recognized. The dominant limitation is texture class, the minor limitations are salinity and alkalinity levels and calcium carbonate contents.

\section{Potential land suitability}

Potential suitability of the studied soils as illustrated in Table 7 and Fig. 3 indicates that the existing two orders $(\mathrm{S})$ and $(\mathrm{N})$ and two classes $\mathrm{S}_{2}$ and $\mathrm{N}_{2}$. The detailed description of these classes is as follows :

$\mathrm{S}_{2}$ : Moderately suitable class represents soils of playa soils (profile 1), peniplain (profile 9) and cultivated plain soils (profiles 3,4,6,8 and 10). The increase in such value is due to the leaching process of salinity and reclamation of alkalinity limitations. Suitability index (Ci) of this class varies from 64.13 to 75.0. Soils of this class have a slight to moderate intensity of texture and slight intensity of calcium carbonate percent. The cost of these land improvements should be taken into account during economic analysis.

\section{$\mathrm{N}_{2}$ : Not suitable}

This suitability class represents the soils of playa (profile 2), Sabkha (profile 5) and sand sheets (profile 7). The suitability index (Ci) of this class is less than 25 . soils of this class have very severe to severe intensity of texture class and slight to severe intensity of soil depth.

The application of chemical and organic fertilizers, green and organic manures and soil conditioners increase the values of capability index. 
TABLE 7. Land suitability classes for the studied soil profiles.

\begin{tabular}{|c|c|c|c|c|c|c|c|c|c|c|c|c|c|c|}
\hline \multirow[t]{2}{*}{$\stackrel{0}{0}$} & \multicolumn{2}{|c|}{$\begin{array}{c}\text { Topography } \\
\text { (t) }\end{array}$} & \multicolumn{2}{|c|}{$\begin{array}{l}\text { Wetness } \\
\text { (w) }\end{array}$} & \multicolumn{4}{|c|}{$\begin{array}{c}\text { Physical } \\
\text { Soil } \\
\text { characteristics }\end{array}$} & \multicolumn{2}{|c|}{$\begin{array}{l}\text { Salinity \& } \\
\text { Alkalinity } \\
\text { (n) }\end{array}$} & \multicolumn{2}{|c|}{$\begin{array}{l}\text { Suitability } \\
\text { index } \\
\text { (Ci) }\end{array}$} & \multicolumn{2}{|c|}{$\begin{array}{l}\text { Suitability } \\
\text { class } \\
\text { (Si) }\end{array}$} \\
\hline & Cs & Ps & Cs & Ps & S1 & S2 & S3 & S4 & Cs & Ps & Cs & Ps & Cs & Ps \\
\hline \multicolumn{15}{|c|}{ Playa } \\
\hline 1 & 100 & 100 & 100 & 100 & 75 & 100 & 95 & 100 & 40 & 100 & 28.5 & 71.25 & $\mathrm{~S} 3$ & $\mathrm{~S} 2$ \\
\hline 2 & 100 & 100 & 100 & 100 & 25 & 55 & 95 & 85 & 80 & 100 & 8.88 & 11.10 & N1 & $\mathrm{N} 2$ \\
\hline \multicolumn{15}{|c|}{ Sabkha } \\
\hline 5 & 100 & 100 & 50 & 100 & 50 & 55 & 95 & 80 & 100 & 100 & 16.36 & 20.9 & N1 & $\mathrm{N} 2$ \\
\hline \multicolumn{15}{|c|}{ Sand Sheets } \\
\hline 7 & 100 & 100 & 100 & 100 & 25 & 90 & 100 & 100 & 58 & 100 & 16.98 & 22.5 & N1 & N2 \\
\hline \multicolumn{15}{|c|}{ Peniplain } \\
\hline 9 & 90 & 100 & 100 & 100 & 75 & 90 & 95 & 100 & 45 & 100 & 25.97 & 64.13 & $\mathrm{~S} 3$ & $\mathrm{~S} 2$ \\
\hline \multicolumn{15}{|c|}{ (Plain) Cultivated area } \\
\hline 3 & 100 & 100 & 100 & 100 & 85 & 90 & 95 & 100 & 98 & 100 & 71.22 & 72.67 & $\mathrm{~S} 2$ & $\mathrm{~S} 2$ \\
\hline 4 & 80 & 100 & 100 & 100 & 85 & 100 & 95 & 90 & 98 & 100 & 56.97 & 72.68 & $\mathrm{~S} 2$ & $\mathrm{~S} 2$ \\
\hline 6 & 100 & 100 & 100 & 100 & 85 & 90 & 95 & 100 & 100 & 100 & 72.62 & 72.68 & $\mathrm{~S} 2$ & $\mathrm{~S} 2$ \\
\hline 8 & 100 & 100 & 100 & 100 & 75 & 100 & 100 & 100 & 58 & 100 & 43.5 & 75.0 & $\mathrm{~S} 3$ & $\mathrm{~S} 2$ \\
\hline 10 & 80 & 100 & 100 & 100 & 75 & 90 & 95 & 80 & 80 & 100 & 29.75 & 64.13 & $\mathrm{~S} 3$ & $\mathrm{~S} 2$ \\
\hline
\end{tabular}

$\mathrm{S} 1=$ Texture, $\mathrm{S} 2=$ Soil depth $(\mathrm{cm}), \mathrm{S} 3=$ Calcium carbonate status and S4 = Gypsum status

$\mathrm{N}=$ suitable, $\mathrm{S} 1$ = High suitability, $\mathrm{S} 2=$ Moderate suitability and S3 = Limitation suitability.

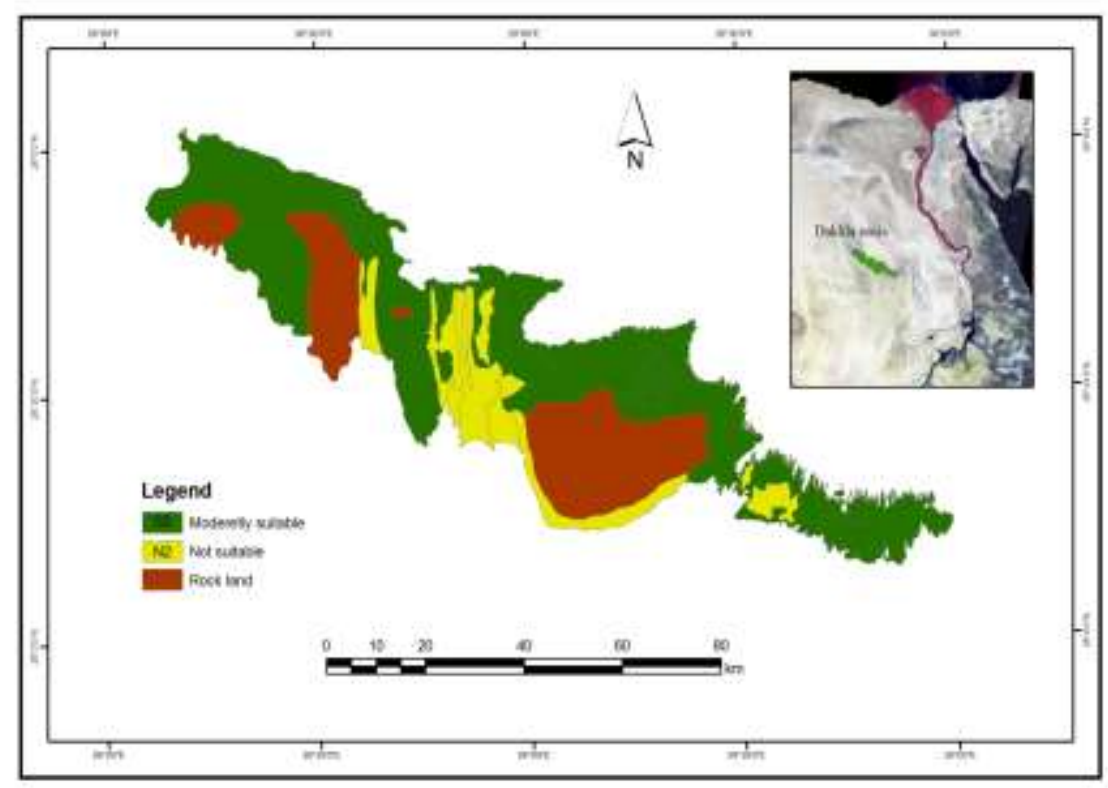

Fig. 2. Current Soil suitability of studied area.

Egypt. J. Soil Sci. 56, No. 1 (2016) 


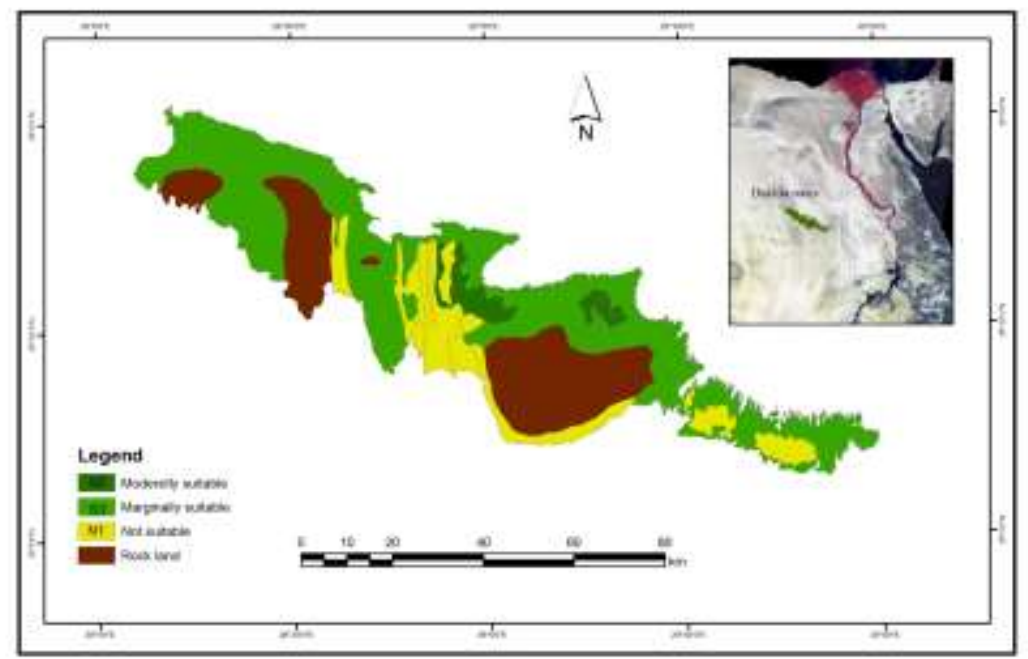

Fig. 3. Potential suitability of the studied area.

Land suitability for specific crops

Land suitability classes for several crops were predicted on the bases of matching land qualities and characteristics and crop standard requirements using the parametric land index as mentioned by Sys et al. (1991 and 1993). The land suitability for selected crops (field crops, vegetables and fruit trees) were investigated. The results of current and potential land suitability are shown in Table 8.

\section{Current suitability}

The results indicate that all the studied soils of playa, sabkha, sand sheet, peniplain and cultivated area (profile 8 and 10) are not suitable for all the studied crops, except for some scattered areas developed on cultivated plain (profile 3,4 and 6) for all the studied crops.

\section{Potential suitability}

1- Playa is moderately suitable $\left(\mathrm{S}_{2}\right)$ for olives and grapes; marginally suitable $\left(\mathrm{S}_{3}\right)$ for wheat, sunflower, groundnut, barley, tomato, potato, green pepper, citrus and palm.

2- Sabkha is highly suitable $\left(\mathrm{S}_{1}\right)$ for groundnut, and not suitable $\left(\mathrm{N}_{2}\right)$ for the rest of crops.

3- Sand sheets are marginally suitable $\left(\mathrm{S}_{3}\right)$ for wheat, tomato, potato, green pepper and citrus; while being not suitable $\left(\mathrm{N}_{2}\right)$ for sunflower, groundnut, barley, onion and palm.

4- Peniplain is highly suitable $\left(\mathrm{S}_{1}\right)$ for potato and grapes; moderately suitable $\left(S_{2}\right)$ for wheat, sunflower, groundnut and olives; marginally suitable $\left(S_{3}\right)$ for barley, onion, tomato, green pepper, citrus and palm.

5- Cultivated plain is highly suitable $\left(\mathrm{S}_{1}\right)$ for groundnut, potato and grapes; moderately suitable $\left(\mathrm{S}_{2}\right)$ for wheat, sunflower, barley, onion, tomato, green pepper, olives and palm; marginally suitable $\left(\mathrm{S}_{3}\right)$ for grapes. 


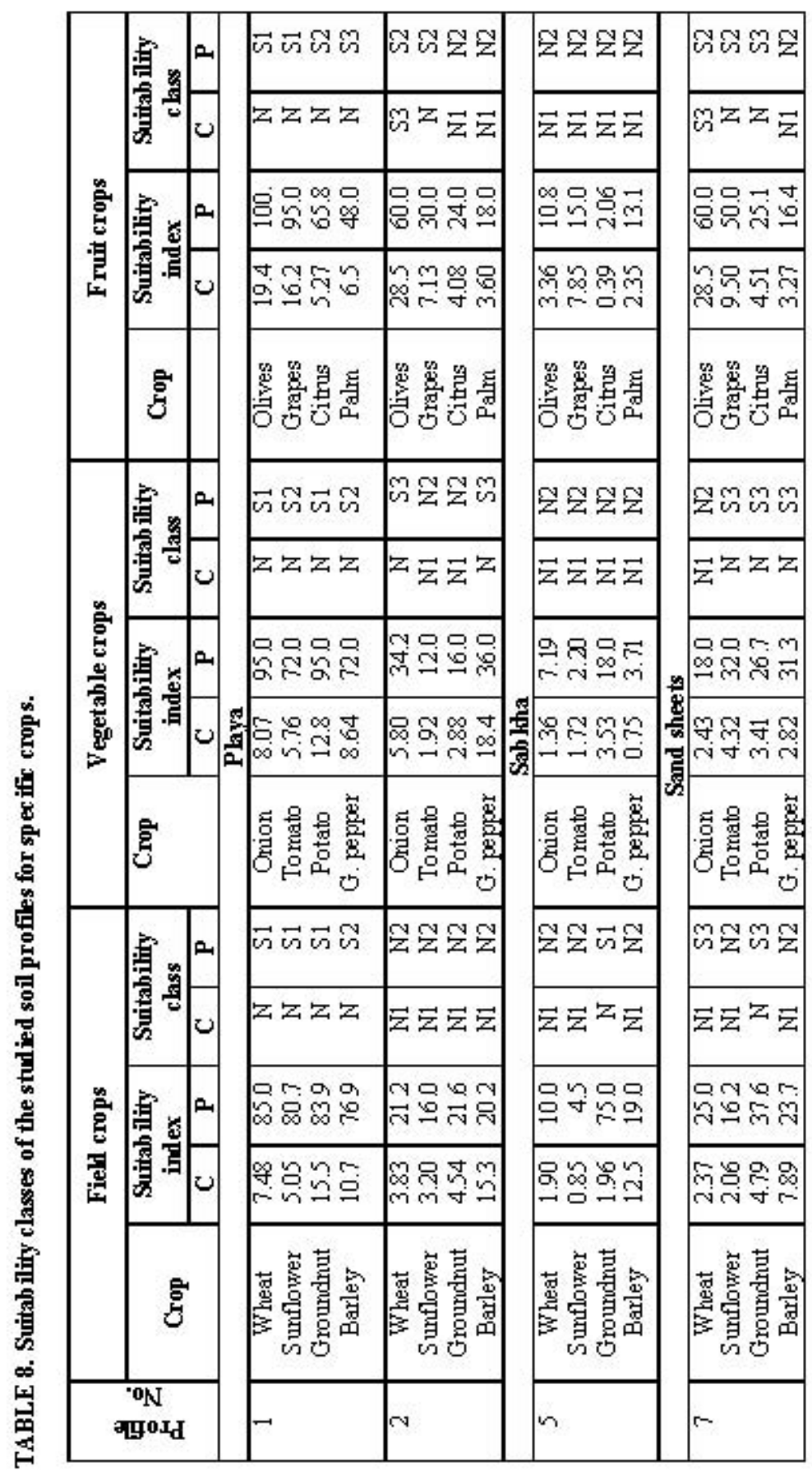




\begin{tabular}{|c|c|c|c|c|c|}
\hline 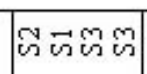 & Иีฒณ & 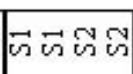 & 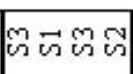 & $\vec{\omega}$ 的园 & ๓ே円 \\
\hline & กิ० & Иேッ⿻ & ஜธะ & 江元完司 & \\
\hline : & 品专它 & & 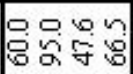 & 象我 & 的 \\
\hline 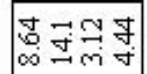 & 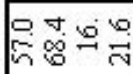 & & 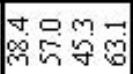 & 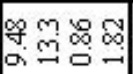 & 过焉 \\
\hline 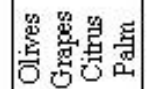 & 总哀 & & 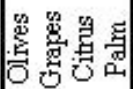 & 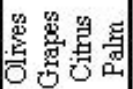 & 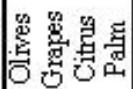 \\
\hline ஜூோ & $\vec{\omega} \circlearrowleft \vec{\sim}$ & พล⿱艹 & $\vec{\omega}$ & $\frac{\mathrm{z}}{\mathrm{z}} \overrightarrow{\mathrm{n}} \mathrm{y}$ & ॠरू \\
\hline 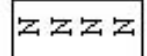 & ॠ & $\mathscr{N}$ & 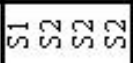 & $\vec{z} \vec{z}{ }^{\prime 2} \vec{z}$ & z北: \\
\hline & & & 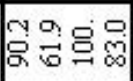 & 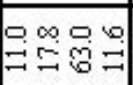 & 要品 \\
\hline & 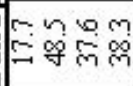 & & & G & $\stackrel{9}{\Rightarrow}=\underset{\infty}{\infty}$ \\
\hline & & & & & 总 \\
\hline ชูกต & $\vec{\omega} \leadsto \vec{n}$ & & : & ถ⿻ & Й \\
\hline 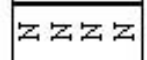 & & 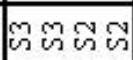 & 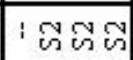 & 江江江 & 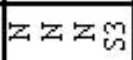 \\
\hline 공잉 & 18. & 8 & 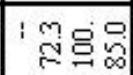 & 8 & 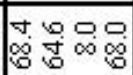 \\
\hline 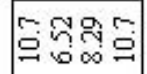 & 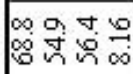 & 高学曼 & 1980 & 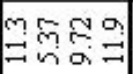 & 5n. \\
\hline 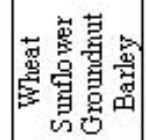 & 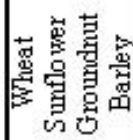 & 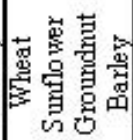 & 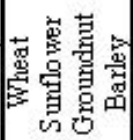 & 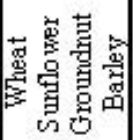 & 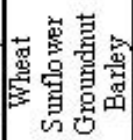 \\
\hline o & , & 寸 & $\circ$ & $\infty$ & 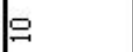 \\
\hline
\end{tabular}




\section{Crop Water requirements}

Table 9 reveals that, the crop water requirements of some selective crops, which are calculated by using climatic data and Crop Wat program. The ETo (evapotranspiration) was estimated using Penman- Monteith equation, after Allen (1998). The crop water requirements were 701.1, 921.0, 740.4, 834.3, 669.0 570.0 640.5, 2085.0, 625.2, 387.6, 1722.0, 1346.1, 373.0, 702.3, 505.2, $595.8,645.3,606.1,466.4$, and $602.1 \mathrm{~mm} / \mathrm{s}$. for tomato (135 day), tomato (180 day) maize grin (125 day), cotton, sorghum, egg plant, peper, banana, flax, barley, citrus 1, citrus 2, pea, peanut, lentil, cucumber, sunflower, onion/ dry, wheat and suger beet, respectively.

Data obtained reveal that consumptive use of crops is considered high, due to the highness of evaporation. It represented with an average of 500, 800 and 1800 $\mathrm{mm} / \mathrm{s}$ for vegetable, field and fruit crops, respectively. So, it is very important to apply suitable irrigation systems such as trickle or sprinkler, where the soil physical properties of the studied physiographic units have good correlation with micron - relief (Zayed and Ashoub , 2000).

\section{Evaluation of irrigation waters sources}

In El- Dakhla Oases, the wells and springs are the main sources for irrigation purposes where the water of the springs that flow to the surface under hydrostatic pressure. Table 10 illustrates irrigation water classification of some ten selected springs and wells at El- Dakhla Oases according to USDA (1991), where $\left(\mathrm{C}_{1}-\mathrm{S}_{1}\right)$ class represents water of West El Mowhob area, El- Kalamon and El- Mowhob village this class of can be used to irrigate most crops in most soils and there are no limiting factors. Water of class $\left(\mathrm{C}_{2}-\mathrm{S}_{1}\right)$ represents the wells and springs of ElZaiate village, Mut, El- Bashandy Village, and El- Mowhob. The class of water is moderately saline and non alkaline. Water of El- Zaiate well (No.10) which is classified as $\left(\mathrm{C}_{3}-\mathrm{S}_{1}\right)$ has high salinity non alkalinity hazards. On the other hand, water of Bathor and El- Zaiate well (No.2) which is classified as $\left(\mathrm{C}_{4}-\mathrm{S}_{4}\right)$ has very high salinity levels and very high alkalinity hazard. This water cannot be used for irrigation of the soils, due to increasing problems of salinity and sodicity in these soils. It is advised to mix the water of El- Zaiate well (No.2) and Bathor spring water with other low salinity and alkalinity values to get over these constraints. 


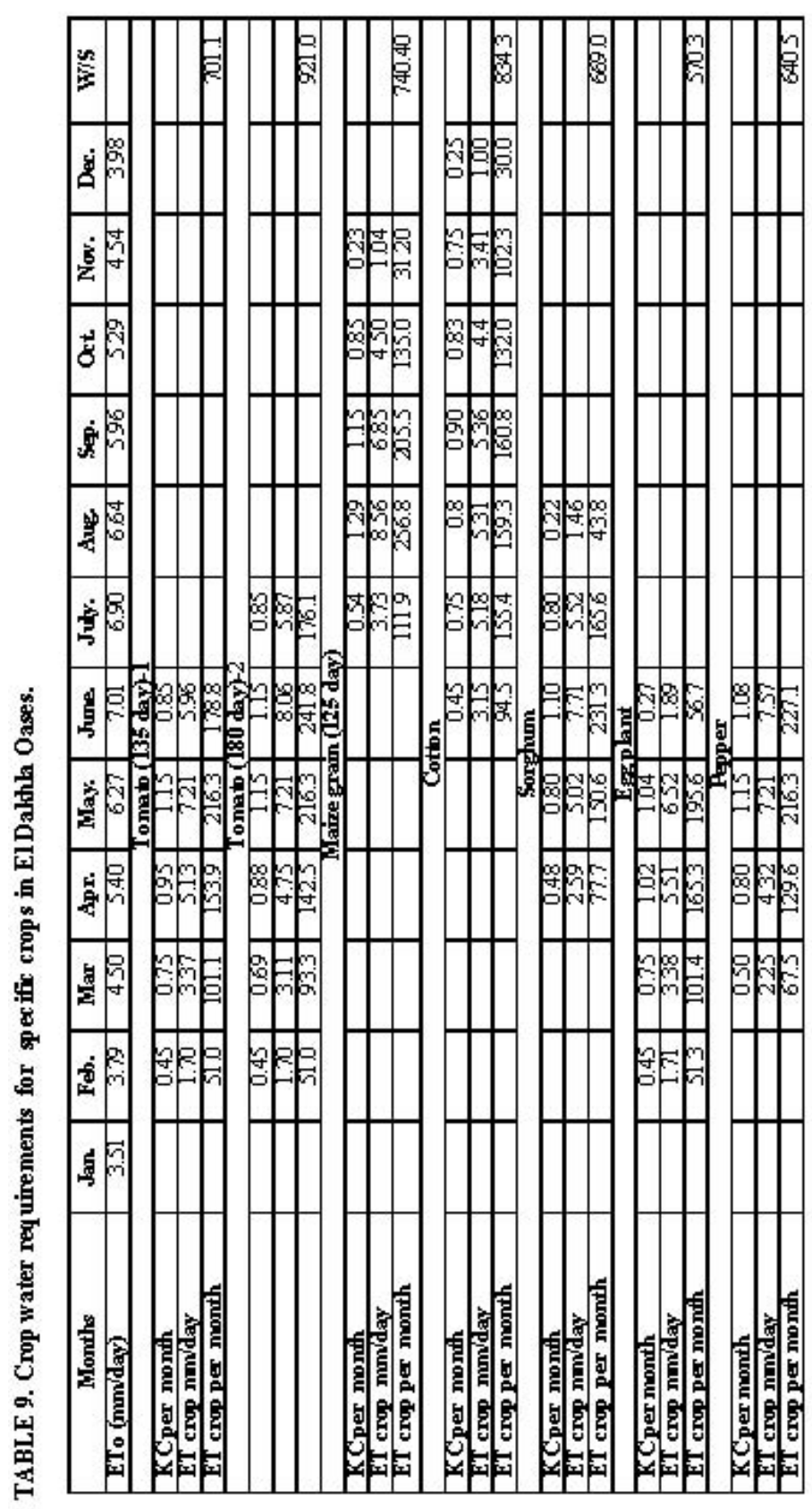

Egypt. J. Soil Sci. 56, No.1 (2016) 


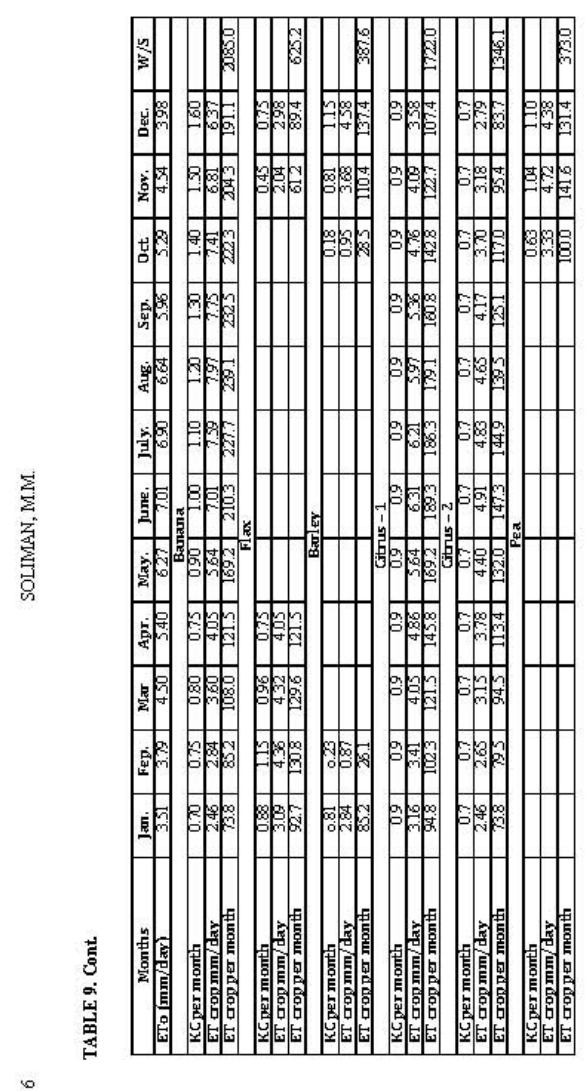

Egypt. J. Soil Sci. 56, No. 1 (2016) 


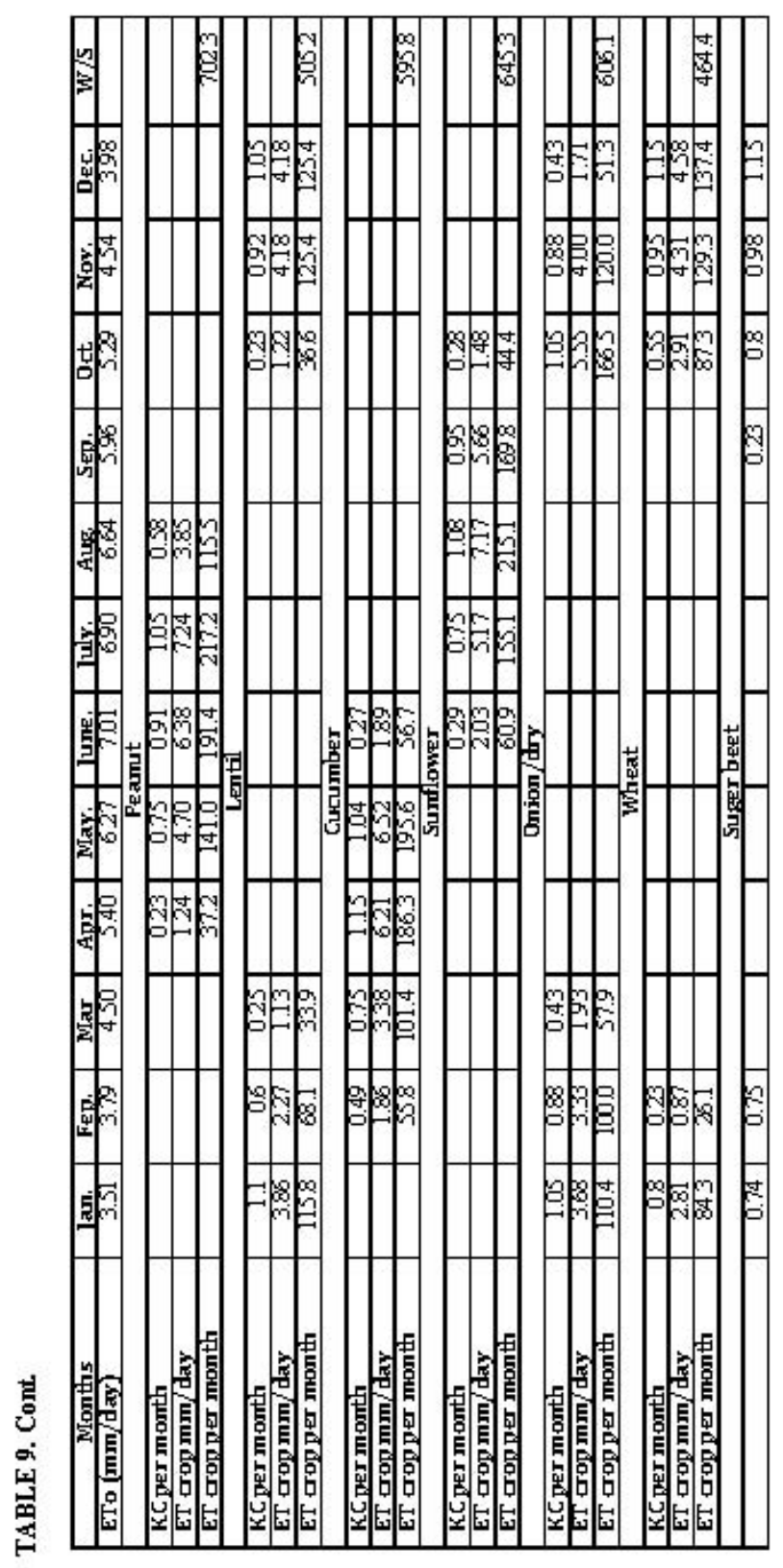

Egypt. J. Soil Sci. 56, No.1 (2016) 


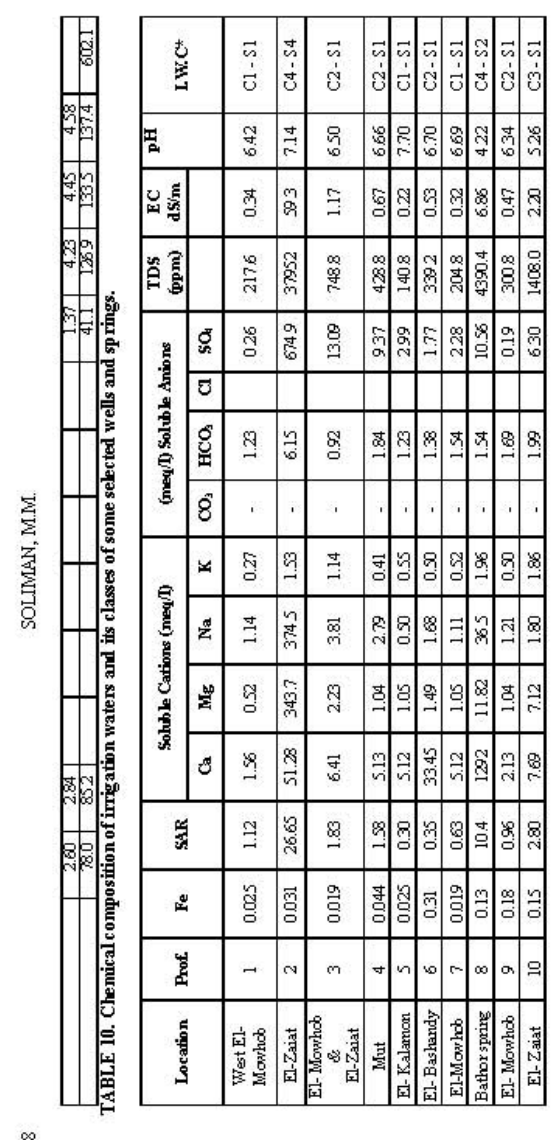

Egypt. J. Soil Sci. 56, No. 1 (2016) 


\section{References}

Abu El-Izz, M.S. (2000) Landforms of Egypt, The American Univ., Cairo Press, Egypt, p. 282.

Allen, R. (1998) "Crop Evapotranspiration Guidelines" for computing crop water requirements, FAO Irrigation and Drainge paper No. 65.Rome, Ittaly.

Attia S.H. (1970) Subsurface Geology of El -Dakhla Oasis. M. Sc. Thesis, Faculty of Science, Cairo University.

CLAC (2010) "Central Laboratory for Agriculture of Climate . Agic. Res. Center, Giza, Egypt.

Egyption Metrological Authority (2010) Climatic Atlas of Egypt ; pubished by Ministry of Transport Arab Republic of Egypt.

FAO (2006) Guideline for Soil Description " $4^{\text {th }}$ ed FAO, Rome, Ittaly.

Folk, R.L. and Ward, W.C.A. (1957) Brazos River bar, a study in the significance of grain size parameters. J. Sed. Perol. 27, 3-8, 3-26.

Hermina, M. (1967) Geology of the nothwestern approaches of Kharga Oasis. Geol. Surv. Cairo, Egypt, 44, 87 p.

Munsell (2010) Munsel Soil Color Charts, Baltimore, Maryland, USA.

Said, R. (2000) The Earth Science, Hurbe and Raw Publishers, New York and London, 814.p.

Said, R. (1962) The Geology of Egypt, Elsevier, Amsterdam, the Netherlands; 377p.

Shata, A.A. (1959) "Geological problems related to the groundwater supply of some desert areas of Egypt”. Bull. Soc. Geogr., J. Egypt, T. 32, 247-263.

Sys, C. (1991) Land Evaluation parts I and II Lecture Notes., Gent. Univ., Gent, Belgium.

Sys, C., Ranst, E., Van Debaveye, J. and Beernaer, T.F. (1993) Land Evaluation part III "Crop Requirements", Agric, Public 7, Gest Adm for Dev. Coop., Brussels, Belgium.

USDA (2004) "Soil Survey Laboratory Methods Manual", Soil Survey Investigation Report No. 42; version 4.0 November, 2004.

USDA (2010) Keys to Soil Taxonomy. $11^{\text {th }}$ Edition. USA.

Zayed, A.M.A. and Ashoub, T.M. (2000) Influence of soil micro - relief on some soil physical properties. Egypt.J. App. Sci., 15 (7), 378-384.

(Received 11/6/2014; accepted 27/12/2014) 


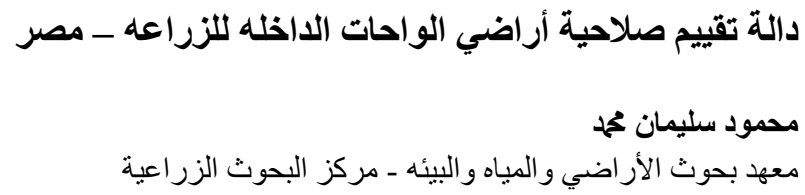

E-mail:mahmoudaldemelawi@hotmail.com :mahmoudeldemelawi@yahoo.com

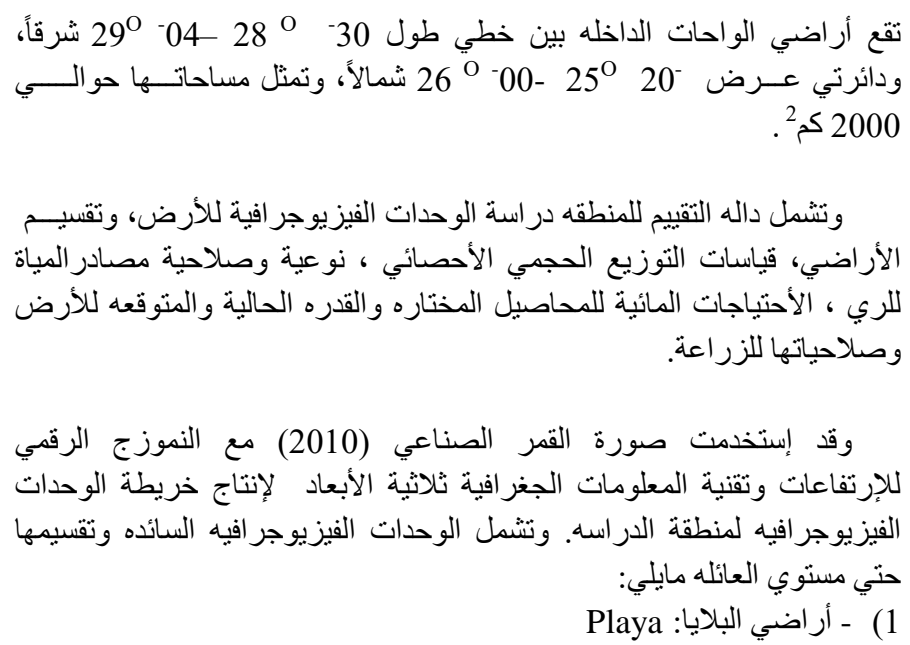

a-Typic Haplosalids, fine loamy over sandy skeletal, mixed, hyperthermic.

b-Duric Haplosalids, sandy skeletal, mixed, hyperthermic.

$$
\text { 2) - أر اضي السبخات: Sabkha }
$$

Lithic Gypsisalids, sandy skeletal, siliceous, Hyperthermic, shallow.

Sand Sheets: أر اضي الفراشات الرمليه:

Calcic Haplosalids, sandy skeletal, mixed, hyperthermic, deep.

$$
\text { 4eniplain : أر اضي أثباه السهول: }
$$

Typic Haplosalids, clayey skeletal, hyperthermic.

Cultivated plain : أراضي الوديان المنزر عه

a- Typic Torriorthents, fine loamy, mixed, hyperthermic.

b-Typic Torriorthents, clayey over fine loamy skeletal, mixed, hyperthermic.

c-Typic Haplosalids, coarse loamy skeletal over clayey, mixed, hyperthermic .

d-Typic Haplosalids, fine loamy over coarse loamy, mixed, hyperthermic. 


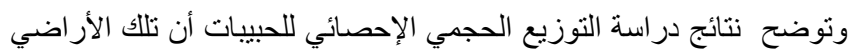

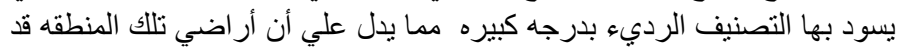

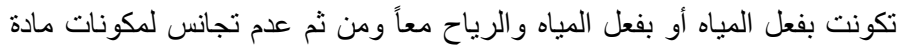

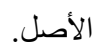

وقد أمكن تحديد القدرة الحاليه لصداحية الأرض للزر اعه الي ثلاث درجات و هي منوسطة الصلاحيه $\left(S_{2}\right)$ - مدية الصلاحية (S)

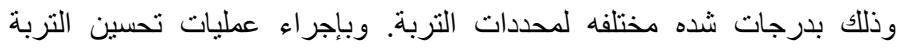

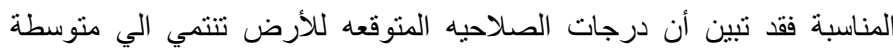
الصلاحية (S $)$ وغير صالحه دائماً (N

وتثير النتائج الي درجة عدم الملائمه الحاليه (N) لزر اعة بعض المحاصيل

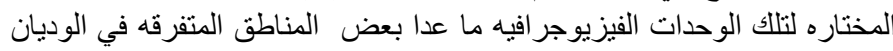

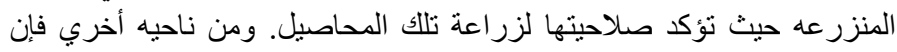

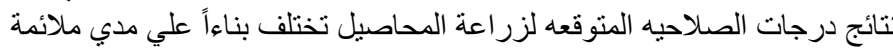
الظروف المتوفره بين خو اص التربة للوحدات الفيزيوجر افيه و الإحتياجات المائيه

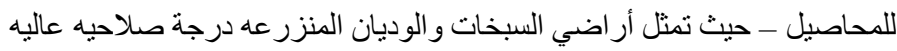

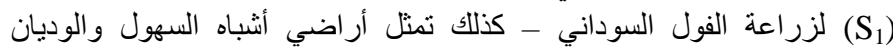

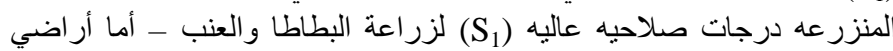

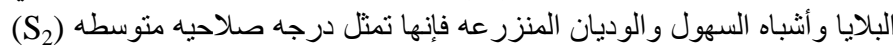

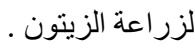

وتعتبرمياه الأبار والعيون الطبيعيه هي المصدر الرئيسي لمياة الري لأراضي

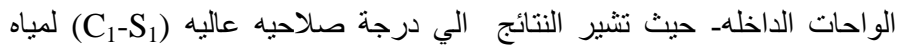

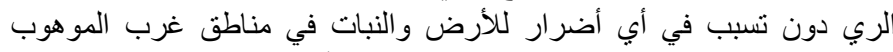
و الكلامون - بينما تمثل درجة صلاحيه منخفضه جداً

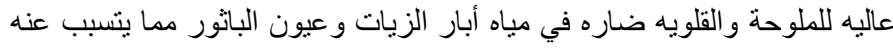

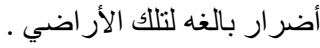

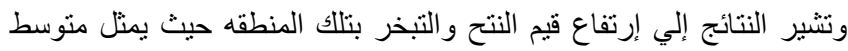

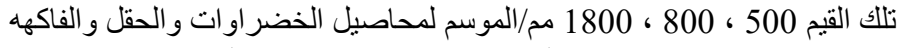

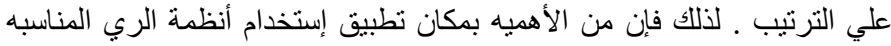

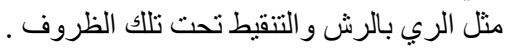

